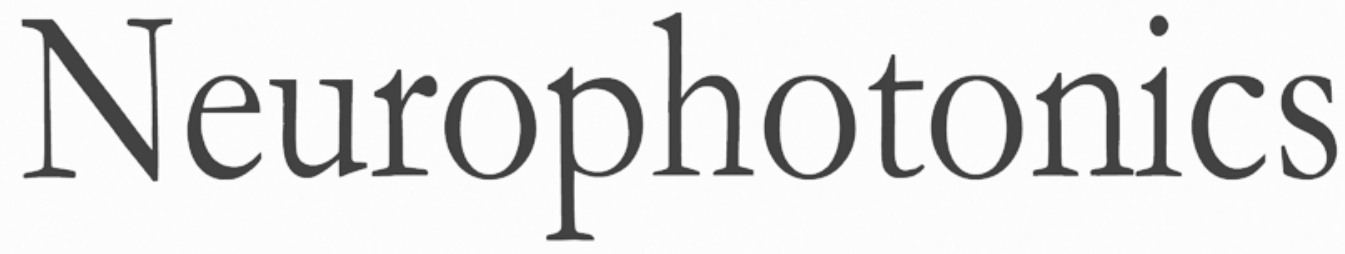

\title{
Exploring attentive task-based connectivity for screening attention deficit/hyperactivity disorder children: a functional near-infrared spectroscopy study
}

Stephanie Sutoko Yukifumi Monden

Tatsuya Tokuda

Takahiro Ikeda

Masako Nagashima

Tsukasa Funane

Hiroki Sato

Masashi Kiguchi

Atsushi Maki

Takanori Yamagata

Ippeita Dan 


\title{
Exploring attentive task-based connectivity for screening attention deficit/hyperactivity disorder children: a functional near-infrared spectroscopy study
}

\author{
Stephanie Sutoko, ${ }^{\mathrm{a}, \mathrm{b}, *}$ Yukifumi Monden, ${ }^{\mathrm{c}, \mathrm{d}}$ Tatsuya Tokuda, ${ }^{\mathrm{b}}$ Takahiro Ikeda, ${ }^{\mathrm{c}}$ Masako Nagashima, ${ }^{\mathrm{c}}$ \\ Tsukasa Funane, ${ }^{a}$ Hiroki Sato, ${ }^{a, \dagger}$ Masashi Kiguchi, ${ }^{a}$ Atsushi Maki, ${ }^{a}$ Takanori Yamagata, ${ }^{c}$ and Ippeita Dan ${ }^{b}$ \\ ${ }^{a}$ Hitachi, Ltd., Center for Exploratory Research, Research and Development Group, Hatoyama, Saitama, Japan \\ ${ }^{\mathrm{b}}$ Chuo University, Research and Development Initiatives, Applied Cognitive Neuroscience Laboratory, Bunkyo-ku, Tokyo, Japan \\ 'Jichi Medical University, Department of Pediatrics, Shimotsuke, Tochigi, Japan \\ IInternational University of Health and Welfare Hospital, Department of Pediatrics, Nasushiobara, Tochigi, Japan
}

\begin{abstract}
Connectivity impairment has frequently been associated with the pathophysiology of attention-deficit/ hyperactivity disorder (ADHD). Although the connectivity of the resting state has mainly been studied, we expect the transition between baseline and task may also be impaired in ADHD children. Twenty-three typically developing (i.e., control) and 36 disordered (ADHD and autism-comorbid ADHD) children were subjected to connectivity analysis. Specifically, they performed an attention task, visual oddball, while their brains were measured by functional near-infrared spectroscopy. The results of the measurements revealed three key findings. First, the control group maintained attentive connectivity, even in the baseline interval. Meanwhile, the disordered group showed enhanced bilateral intra- and interhemispheric connectivities while performing the task. However, right intrahemispheric connectivity was found to be weaker than those for the control group. Second, connectivity and activation characteristics might not be positively correlated with each other. In our previous results, disordered children lacked activation in the right middle frontal gyrus. However, within region connectivity of the right middle frontal gyrus was relatively strong in the baseline interval and significantly increased in the task interval. Third, the connectivity-based biomarker performed better than the activation-based biomarker in terms of screening. Activation and connectivity features were independently optimized and cross validated to obtain the best performing threshold-based classifier. The effectiveness of connectivity features, which brought significantly higher training accuracy than the optimum activation features, was confirmed ( $88 \%$ versus $76 \%$ ). The optimum screening features were characterized by two trends: (1) strong connectivities of right frontal, left frontal, and left parietal lobes and (2) weak connectivities of left frontal, left parietal, and right parietal lobes in the control group. We conclude that the attentive task-based connectivity effectively shows the difference between control and disordered children and may represent pathological characteristics to be feasibly implemented as a supporting tool for clinical screening. () The Authors. Published by SPIE under a Creative Commons Attribution 4.0 Unported License. Distribution or reproduction of this work in whole or in part requires full attribution of the original publication, including its DOI. [DOI: 10.1117/1.NPh.6.4.045013] Keywords: attention-deficit/hyperactivity disorder; near-infrared spectroscopy; task-based connectivity; attention; screening tool.
\end{abstract}

Paper 19068R received Jul. 2, 2019; accepted for publication Nov. 20, 2019; published online Dec. 17, 2019.

\section{Introduction}

Attention-deficit/hyperactivity disorder (ADHD), which is characterized by symptomatic inattention and hyperactivity/ impulsivity, is diagnosed in early childhood to adolescence, ${ }^{1}$ and ADHD is labeled as one of the most prevalent neurodevelopmental disorders. ${ }^{2}$ When Faraone et al. ${ }^{3}$ suggested comparable prevalence rates across populations and argued against an ADHD hypothesis as a cultural construct, ${ }^{4}$ Polanczyk et al. ${ }^{5}$ suggested that geographical differences in prevalence rates are caused by the methodological characteristics rather than the associated perspective of culture. Despite the common application of standard guidelines [e.g., the Diagnostic and Statistical Manual of Mental Disorders, Fifth Edition (DSM-5) and the International Classification of Diseases, Eleventh

\footnotetext{
*Address all correspondence to Stephanie Sutoko, E-mail: stephanie.sutoko tc@ @itachi.com

${ }^{\dagger}$ Present address: Shibaura Institute of Technology, Department of Bioscience
} and Engineering, Minuma-ku, Saitama, Japan
Revision], discrepancies in diagnoses based on the guidelines were found. ${ }^{6,7}$ These findings have triggered the need for a paradigm shift from behavioral-based assessment to biomarker evaluation for clinically supporting more objective diagnosis and even prognosis. ${ }^{8,9}$

Genetic and molecular markers have been investigated; ${ }^{10-13}$ however, invasive intervention (e.g., drawing blood) might hinder the clinical practicability of those biomarkers for longitudinal prognostic monitoring in particular. As we come to understand the pathophysiology underlying ADHD, the relationship between causal variables and disease outcomes can be clearly interpreted and suggests the use of more valid biomarkers. ${ }^{9}$ Even though confounding and diverse environmental factors should not be neglected, due to abnormal cognitions and behaviors, ADHD and other neuropsychiatric disorders are frequently associated with brain impairments due to abnormal cognitions and behaviors. ${ }^{14}$ Therefore, neuroimaging-based biomarkers, with advantages in understanding brain structures, functions, and networks, have recently gained popularity. ${ }^{15,16}$ 
As a neuroimaging technique with high spatial resolution, functional magnetic-resonance imaging (fMRI) provides abundant information about brain features, including anatomical structure (e.g., cortical thickness, surface area, and volume), activation, and networks [e.g., functional connectivity (FC), graph-theory parameters, and regional homogeneity]. ${ }^{17,18}$ The prominence of $\mathrm{FC}$ among other features in diagnosing ADHD had been evidenced. ${ }^{19} \mathrm{~A}$ high discrimination rate $(80 \%$ to $86 \%)$ between ADHD and healthy children and adults was demonstrated on the basis of resting-state (RS) fMRI data. ${ }^{20}$ To develop accurate and robust diagnostic biomarkers, large and aggregate ADHD datasets (containing characteristic and RS fMRI data) with sample numbers greater than 900 subjects have been collected. ${ }^{21}$ Depending on the selected features and classifiers, classification accuracy for typically developing (TD) and (subtypes) ADHD children was widely distributed (i.e., respectively, $37.44 \%$ to $60.51 \%$ and $49.79 \%$ on average). On the other hand, classification accuracy was improved (to $62.52 \%$ ) by excluding imaging data and solely using characteristic data such as age, gender, handedness, and full-scale intelligence quotient (IQ). ${ }^{22}$ The robustness of RS connectivity features was then argued. The lack of robustness might be caused by the low quality of measurements on children ( $30 \%$ to $50 \%$ failure rate) due to motion artifacts. ${ }^{23,24}$

Functional near-infrared spectroscopy (fNIRS) was introduced more than 20 years ago ${ }^{25}$ to measure the change in concentration of cerebral hemoglobin (which is closely related to the brain's metabolic activity). ${ }^{26}$ By offering higher temporal resolution, better motion tolerance, and less measurement burden on claustrophobic subjects than fMRI, fNIRS is feasible for measurements on children. Due to moderate spatial resolution and the limited probe number of fNIRS, activation analysis was likely more approachable than network analysis. However, due to the demand of subject-friendly measurements for broader populations (i.e., infants and children) and clinical purposes, the potential of network analysis using fNIRS was thoroughly investigated. High similarity between RS FCs of bilateral primary motor regions obtained from simultaneous fMRI and fNIRS measurements has been reported. ${ }^{27}$ Moreover, the intermodal similarity in diverse regions was evaluated, and a high correlation between fNIRS and fMRI signals (even in the distant regions as incorporated within an RS network) was shown in Ref. 28. In addition, reliability and reproducibility of multiple intrasubject measurement ${ }^{29,30}$ and various instrumentations ${ }^{31}$ were assessed. These studies provide prominent evidence of the usability of fNIRS in regard to network analysis. ${ }^{32}$ Despite having advantages of less task demand and valuable FC information, the RS task encounters problems related to test-retest reliability. ${ }^{33}$ Asking subjects to perform certain tasks may minimize uncontrollable variances; however, only a limited number of task-based connectivity studies have been done.

Activation analysis based on fNIRS measurement of TD and ADHD populations has been examined under various task performances (e.g., inhibition control, attention response, and verbal fluency). ${ }^{34-37}$ According to those measurements, ADHD children showed significantly less activation in task-dependent regions of interest (ROIs). For example, higher right inferior frontal gyrus/middle frontal gyrus (IFG/MFG) and bilateraltemporal activations were observed in TD children during inhibition $^{38}$ and facial expression ${ }^{39}$ tasks, respectively. We are currently interested in investigating the differences in attentive FCs based on fNIRS measurements on TD and ADHD children during the visual-oddball (OB) tasks. Analysis of FC is expected to be crucial in explaining the relationships between cognitive functions, ROIs [e.g., right IFG/MFG and right supramarginal gyrus (SMG)/angular gyrus (ANG)], ${ }^{35,40}$ and networks. Furthermore, the association between FCs and activation was evaluated. As RS networks are known to be dynamic and time varying, ${ }^{41-43}$ task-evoked FC might be anticipated as being distinct, namely, in between task transitions (i.e., baseline and task). Furthermore, the feasibility of task-based FC for clinical screening biomarker was explored, assessed, and compared to the activation biomarker.

\section{Materials and Methods}

\subsection{Data Acquisition}

Thirty-seven children, who met the ADHD criteria specified in DSM-5, participated in the experiment on attention control. The children were nonmedicated naïve, and among them, 22 (19 boys; $9.5 \pm 2.0$ years old) and 15 (12 boys; $9.9 \pm 2.1$ years old) were prescribed methylphenidate (MPH) and atomoxetine (ATX), respectively. Autism spectrum disorder (ASD) symptoms were observed in 21 of the ADHD children, and they were designated as ASD-comorbid ADHD children. One of the children, a 9-year-old girl, was excluded from the analysis due to her comorbidity condition with epilepsy. Both the ADHD children and the ASD-comorbid ADHD children were defined as the disordered group. Furthermore, 23 TD children (15 boys; $9.8 \pm$ 1.9 years old) joined the experiment as the control group. The full-scale IQ of all subjects was assessed according to the Wechsler Intelligent Scale of Children-Third Edition. All subjects received detailed prior explanations, and their guardians signed their informed consent. The subjects were subjected to fNIRS measurements by clinical doctors at Jichi Medical University Hospital (Tochigi, Japan) with the approval of the Ethics Committee of Jichi Medical University Hospital. The measurement data were obtained according to the regulations of the Internal Review Board of the Central Research Laboratory, Hitachi, Ltd. and Chuo University. The current data originally came from two datasets-MPH- and ATX-prescribed ADHD datasets. These datasets had been used, analyzed, and reported, either completely or partly, in our previous publications with different purposes (i.e., brain activation in group analysis and development of a signal processing method). ${ }^{35,40,44}$

\subsection{Experimental Design and Measurement}

The experimental design was based on a double-blind, placebocontrolled, cross-over study. It entailed two measurement sessions (4 to 30 days apart) under pre- and postadministration conditions in each session. The order of medication and placebo administration for each subject was randomized. Because all the disordered-group children were nonmedicated naïve, any preadministration conditions were imposed after the subjects were not given their medications for 2 to 4 days (i.e., wash out). The effect of the medication or placebo was evaluated $1.5 \mathrm{~h}$ after administration. The current study aims to develop a screening biomarker; therefore, only the preadministration data of the first measurement session in the disordered group were used in the current classification analysis. Neither the medication nor the placebo was administered to the control group. This setup resulted in a single-time measurement for each TD child. 
An attention task, the OB task, was performed. The OB task required the subjects to respond by pressing one of two (blue or red) buttons when shown a specific visual stimulus (e.g., pictures of animals). There were two stimulus types: standard and target. The occurrence of target to standard stimulus was equivalent to the ratio 1:4. To create a baseline (i.e., control condition), subjects were asked to react in the same manner (e.g., always press the blue button) regardless of the visual stimulus. The frequency at which the stimulus was displayed was $1 \mathrm{~Hz}$; accordingly, both baseline and task intervals lasted for $25 \mathrm{~s}$. The total measurement time was about 6 to 7 min with six and seven repetitions of task and baseline intervals, respectively. The experimental design is explained in more detail elsewhere. . $^{35,40,44}$

Behavioral performances have been previously reported. ${ }^{35,40}$ The control group revealed a lower error of omission (i.e., subject failed to press any button when target stimuli were displayed) than the disordered group (two-sample $t$-test; $p<0.05$; degree of freedom $(\mathrm{DF})=28$ to 42 ). Furthermore, the reaction time of correct response was faster in the control group (two-sample $t$-test; $p<0.05 ; \quad \mathrm{DF}=28$ to 42 ). However, there was no significant difference in terms of error of commission (i.e., subjects failed to press a correct button when target stimuli were displayed) between the control and disordered groups (two-sample $t$-test; $p \geq 0.05$; $\mathrm{DF}=28$ to 42 ). The effects of medication on behavioral performance have also been previously described in detail. ${ }^{35,40}$

A dual-wavelength fNIRS system (ETG-4000; wavelengths: 695 and 830 nm; Hitachi Medical Corporation, Tokyo, Japan) was used for measuring the change of cerebral hemoglobin during the OB task. A $3 \times 5$ plane probe (consisting of eight emitters and seven detectors) was used. The measured region was approximately located between the emitter and the detector (separated by a distance of $3 \mathrm{~cm}$ ). Two plane probes were placed on the bilateral prefrontal-to-parietal cortices, which were covered by 44 channels in accordance with the procedure described by Nagashima et al., ${ }^{35,40}$ as shown in Fig. 1. Three-dimensional (3-D) channel spaces were digitized and then spatially registered on the standard Montreal Neurological Institute atlas. ${ }^{45,46}$

\subsection{Signal Preprocessing and Feature Extraction}

fNIRS measurement data were preprocessed on the MATLAB ${ }^{\circledR}$ based platform (platform for optical topography analysis tools). ${ }^{47}$ The change in transmitted light intensity was converted into signals reflecting changes in concentrations and optical path length $(\Delta C \cdot L)$ of oxygenated, deoxygenated, and total hemoglobin $\left(\mathrm{O}_{2} \mathrm{Hb}, \mathrm{HHb}\right.$, and $\mathrm{Hb}$-total $)$ using the modified Beer-Lambert equation. ${ }^{26,48}$ Channel-wise signals with low quality (signal-to-noise ratio $<10 \mathrm{~dB}$ ) were eliminated. Channels with a high number of time-point data (outliers across channels) affected by extremely high- or low-signal amplitudes (outlier amplitudes; $\Delta C \cdot L>\mu+3 \sigma \vee \Delta C \cdot L<\mu-3 \sigma ; \mu$ and $\sigma$ are average and standard deviation of all channels, respectively) were also rejected. Due to these criteria, $12 \%$ to $12.5 \%$ of total channels were eliminated. The remaining channel-wise signals were linearly fitted and filtered (finite impulse response: 0.01 to $0.8 \mathrm{~Hz}$ ) to remove disruptive baseline and cardiac pulsation. Thereafter, activation and FC analyses were applied on filtered signals.

\subsubsection{Activation analysis}

Continuous signals were compartmentalized on the basis of 13 , 25 , and $13 \mathrm{~s}$ for pretask, task, and posttask, respectively [Fig. 2(a)]. Therefore, six epochs with shorter temporal signals (51 s) were obtained from a continuous channel-wise signal. Motion artifacts might uncontrollably distort the epoch data, so motion-affected epochs were rejected. Specifically, the occurrence of motion artifacts (e.g., spikes) was recognized by the interepoch dissimilarity in the channel data. ${ }^{44}$ The dissimilarity was quantified using the summation of interepoch correlations. Compared to other epochs (which can be caused by motion artifacts), epochs with significantly low-correlation summation (nonparametric outlier; \pm 3 interquartile range) are likely to have distinct waveforms. ${ }^{49}$ Those epochs were then excluded from further analysis. An epoch was rejected in maximum $[6.86 \%$ of total channels; Fig. 2(b)]. Epoch rejection was uniform for $\mathrm{O}_{2} \mathrm{Hb}$ and $\mathrm{HHb}$. The baseline of motion-free epochs was corrected to eliminate redundant drifts (i.e., the first $10 \mathrm{~s}$ of the epochs). Subsequently, motion-free epochs were averaged for each channel [Fig. 2(c)]. The activation analysis was performed by averaging the epoch data $\left(\mathrm{O}_{2} \mathrm{Hb}\right.$ and $\left.\mathrm{HHb}\right)$ from 17 to $38 \mathrm{~s}$ [dark yellow-colored interval in Fig. 2(c)].

\subsubsection{FC analysis}

Rejecting motion-affected epochs and averaging motion-free epochs accordingly minimizes noises and improves data quality
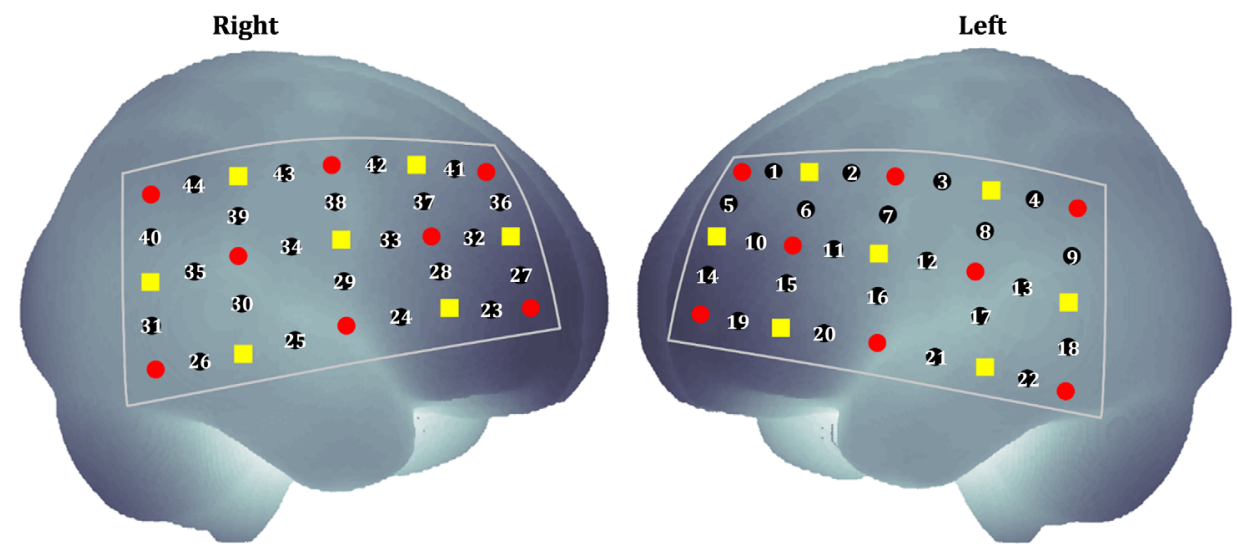

Fig. 1 Probe placement on bilateral hemispheres. A plane probe consisted of eight emitters (red circles) and seven detectors (yellow squares). Two plane probes measured 44 regions, namely, channels (numbered black circles). A channel was approximately located between an emitter and a detector that were separated by $3 \mathrm{~cm}$. 

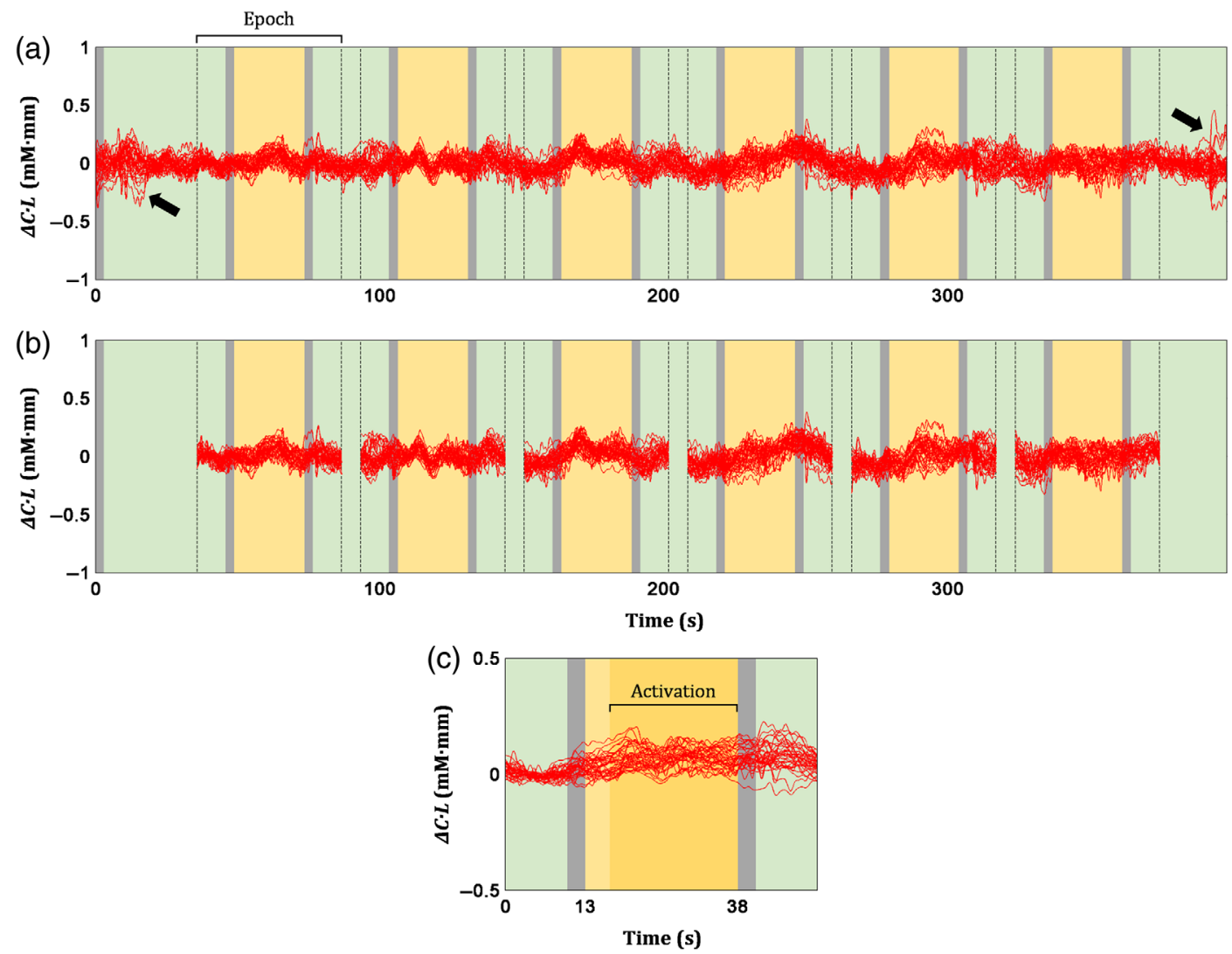

Fig. 2 Signal preprocessing for activation analysis. (a) Continuous signals (red plots; all channels) were divided into six shorter signals, namely, epochs. An epoch data included 13, 25, and 13 s of pretask, task (yellow-colored), and posttask intervals, respectively. Spikes were detected at the beginning and end of measurement (black arrows). (b) Furthermore, motion-affected epochs were identified and rejected in a channel-wise manner. (c) Motion-free epochs were then averaged for each channel. Channel-wise activation values were obtained by averaging the epoch data from 17 to $38 \mathrm{~s}$ (dark yellow-colored interval). Green- and gray-colored intervals indicate baseline and instruction intervals.

[Fig. 2(c)]. However, that method is impractical for FC analysis because the temporal information will be neglected. Therefore, instead of epoch data, time-point data affected by motion artifacts (e.g., spikes) were eliminated. Spikes were detected by the sudden change $(>0.1 \mathrm{mM} \cdot \mathrm{mm})$ in two timepoint data $(0.1 \mathrm{~s})$ and removed from continuous signals [Fig. 3(a)]. Extremely high- or low-signal amplitudes were frequently observed pre- and postspikes. Therefore, time-point data with outlier amplitudes were also eliminated [Fig. 3(b)]. Rejection of time-point data was uniform for $\mathrm{O}_{2} \mathrm{Hb}$ and $\mathrm{HHb}$ $(24.0 \% \pm 14.6 \%)$. To understand the dynamics of connectivity, the continuous signal was temporally categorized into baseline (green-colored) and task (yellow-colored) intervals. Those interval signals were then concatenated for each category [Figs. 3(c) and 3(d) for baseline and task, respectively]. ${ }^{50}$ Interchannel connectivity was evaluated using Pearson's correlation $(r)$ for the entire measurement, baseline, and task intervals of $\mathrm{O}_{2} \mathrm{Hb}$ and $\mathrm{HHb}$ signals.

\subsection{Classification Analysis}

Both activation and connectivity magnitudes were treated as potential classification (i.e., screening) features. Because lowquality and noisy data were excluded, the number of available features for each subject might be varied. The availability of features should be sufficient and balanced in both control and disordered groups. Therefore, features with low availability $(<50 \%)$ and high discrepancy of group availability $(>10 \%)$ were disregarded in the following classification analysis.

The classification analysis was performed and optimized following the process flow summarized in Fig. 4. Because the sample number in this study was limited compared to the number of maximum available features $(44 \times 43 / 2$ and 44 for connectivity and analysis features, respectively), the risk of overfitting and the curse of dimensionality might be elevated. Therefore, feature selection and cross-validation analysis were also accommodated in this classification analysis to avoid those risks. Features were selected according to the stepwise-forward approach, namely, adding features one-by-one. ${ }^{51}$ Exhaustive feature selection (i.e., all possible feature combinations) might be beneficial in regard to classification performance; however, the computational training time would be impractical, particularly in the case of connectivity features. By considering the trade-off between computational training time and classification performance, we chose the stepwise-forward selection. Features were also cross validated by the $k$-fold (fivefold) cross-validation method (approx. 1:2 ratio of control to disordered subjects in both training and test subsets).

For the first step, a single feature [Fig. 4(a)] was statistically evaluated (two-sample $t$-test) in order to clarify the feature trend for each group in the training subsets (e.g., the control group tended to exhibit greater feature magnitude than the disordered 

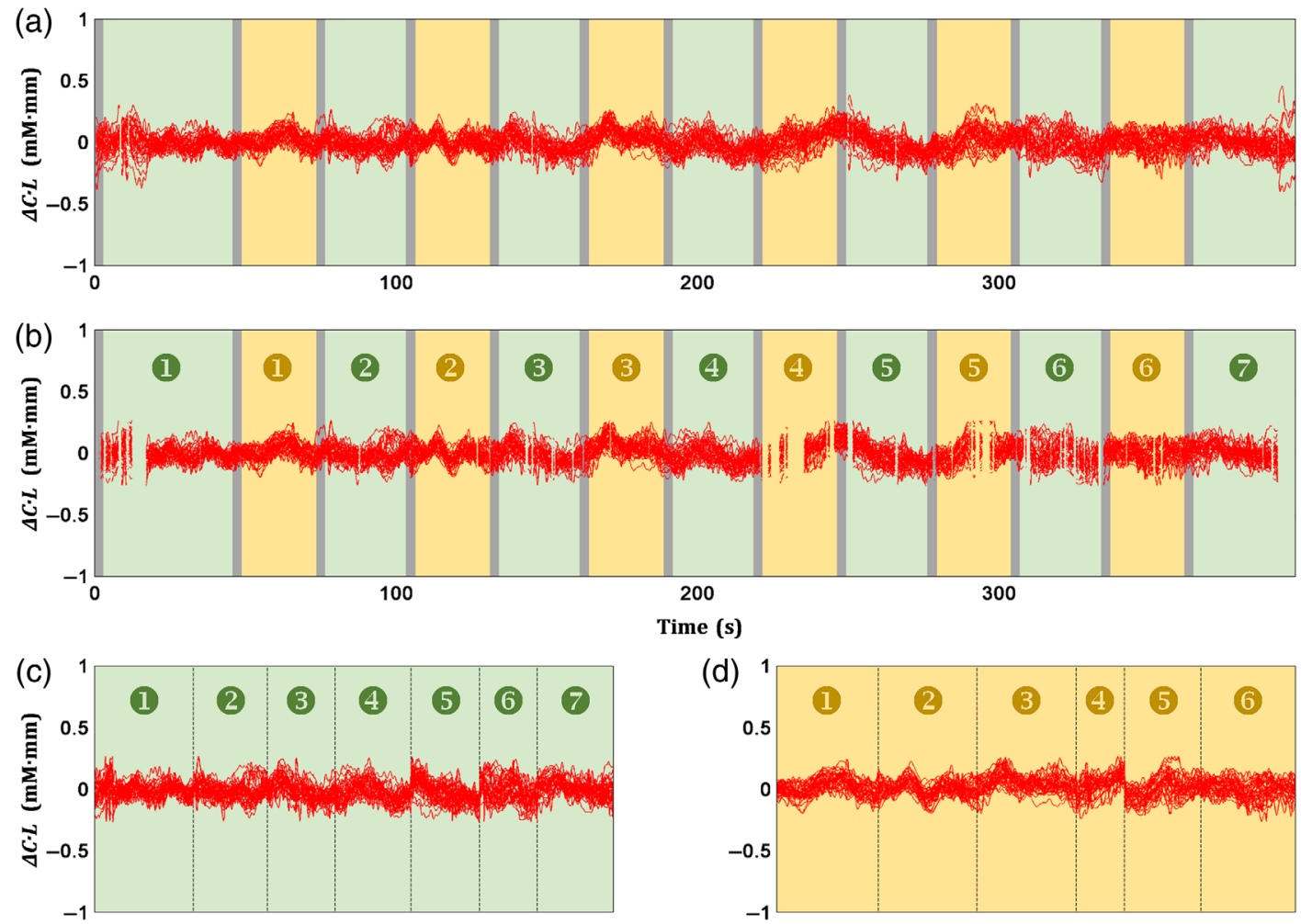

Fig. 3 Signal preprocessing for connectivity analysis. (a) Sudden amplitude changes ( $>0.1 \mathrm{mM} \cdot \mathrm{mm}$; spikes) in two time-point data $(0.1 \mathrm{~s})$ were detected and rejected from the continuous signals (red plots). Motion artifacts also affected pre- and postspikes resulting in extremely high- or low-signal amplitudes (i.e., outlier amplitudes). (b) Time-point data with outlier amplitudes were also eliminated. (c) Seven baseline (green-colored intervals) and (d) six task (yellow-colored intervals) signals were concatenated. Graycolored intervals indicate instruction intervals.

group) [Fig. 4(b)]. Supervised classification analysis was performed afterward by varying the thresholds of feature magnitude (e.g., in steps of 0.001) and discriminating subjects according to feature trends (e.g., subjects with feature magnitudes greater than a threshold were classified as the control group, and vice versa) [Fig. 4(c)]. Specificity (i.e., the true control subjects) and sensitivity (i.e., the true disordered subjects) were then computed. The thresholds giving the highest summation of specificity and sensitivity [Fig. 4(d)] were then used to evaluate the test subsets [Fig. 4(e)]. This process was repeated for any other single feature. Among all features, the feature that contributed to the highest summation of cross-validated and averaged accuracies in both training and test subsets was selected [Fig. 4(f)]. Classification accuracy is defined as the correct classification rate from the total number of subjects in a subset. For the second step, the previously selected feature was combined with another single feature [Fig. 4(g)]. If two or more features were combined, those features would be averaged. Averaged connectivity is defined as the inverse of averaged Fisher- $z$ transform. Averaged features were used to classify the training subsets. Validation was subsequently carried out in the test subsets. All possible combinations of two features involving the previously selected feature were evaluated. Among all combinations, the two-feature combination giving the highest summation of cross-validated and averaged accuracies in both training and test subsets was selected. The third step aimed to find the best performing combination of three features and so on.

The computation for adding features one-by-one was continuously done. In order to minimize computational time, when the stepwise summation of training and test accuracies was $10 \%$ lower than the highest accuracy as currently obtained [5\% of significance level for each of the training and test accuracies; Fig. 4(h)], the computation would be terminated [Fig. 4(i)]. Optimum features are defined as the combination of features giving the highest summation of training and test accuracies. Classification performance in terms of connectivity and of activation features was then compared to evaluate the potential of the screening biomarker.

In obtaining the best classification performance, the effectiveness of the stepwise method was evaluated and compared to three others: best-performing single, significant betweengroups, and all available features. The selected feature at the first step of stepwise classification was used in the method of best performing single feature. Meanwhile, significant features with the major between-group trend (e.g., significantly higher activation or stronger connectivity in the control group, and vice versa) were selected for the method of significant betweengroup features. For a standard comparison, no feature selection (i.e., usage of all features) was applied on the method of all available features.

\section{Results}

\subsection{Group Effect in Functional Connectivity}

Subject-averaged connectivity (Fisher- $z$ transform) in the entire measurement, baseline, and task intervals is shown in Fig. 5. $\mathrm{O}_{2} \mathrm{Hb}$ connectivities [Figs. 5(a) and 5(b)] were well correlated $(r \geq 0.7$; Fig. $\mathrm{S} 1$ in the Supplemental Material) to $\mathrm{HHb}$ 


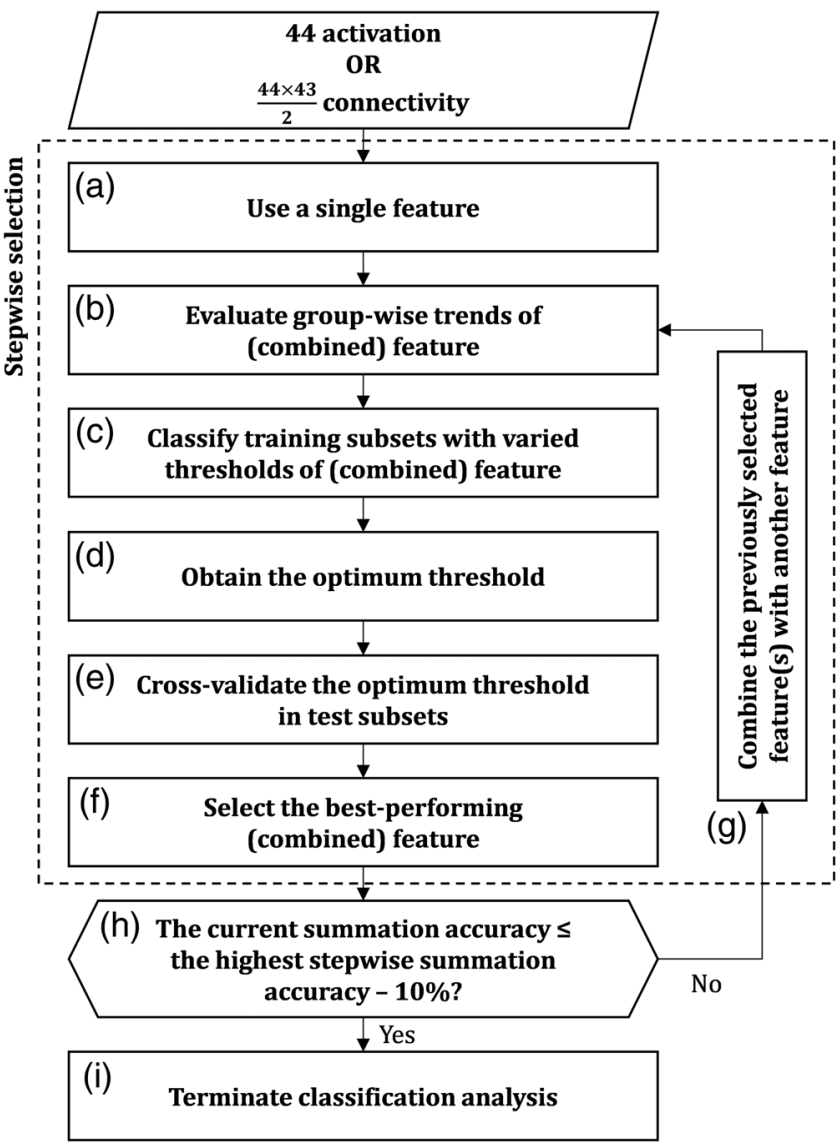

Fig. 4 Process flow of classification analysis. The classification analysis was carried out independently for each activation and connectivity feature. For the first step, (a) a single feature was used to classify groups. (b) The feature was statistically evaluated to understand group-wise trends. (c) The thresholds of feature were varied and applied on the training subsets. (d) Selection of optimum threshold was done based on the highest summation of specificity and sensitivity. (e) The optimum threshold was cross validated in the test subsets. (f) The best performing feature with the highest summation of cross-validated and averaged accuracies in both training and test subsets was carried on to the succeeding stepwise selection. In the second step, (g) the best performing combination of two features was selected using the same procedure (b)-(f). Therefore, the selected features would be added one-by-one for each step. (h) If the trend of summation accuracy was decreasing, (i) the classification analysis would be terminated.

connectivities [Figs. 5(c) and 5(d)]. During the baseline interval [Figs. 5(a2)-5(d2)], within region connectivity of $\mathrm{O}_{2} \mathrm{Hb}$ and $\mathrm{HHb}$ was observed in the right MFG for both the control and disordered groups. The control group showed slight increases in interhemispheric connectivity during the task [Figs. 5(a3) and $5(\mathrm{c} 3)$ ]. Meanwhile, bilateral intra- and interhemispheric connectivities were found to increase during the task performed in the disordered group [Figs. 5(b3) and 5(d3)]. Unaffected within region connectivity of the right MFG was observed during the task in the control group. Significant task-evoked increases of connectivity were observed in the disordered group. This result may suggest that the control group relatively maintained the attentive connectivity, even in the baseline interval. Statistical results of interinterval [one-sample $t$-test; $p<0.05$ (uncorrected); $\mathrm{DF}=10$ to 22 for the control group; $\mathrm{DF}=17$ to 35 for the disordered group; DF depends on each feature availability] are shown in Fig. S2 in the Supplemental Material.
Between-group differences in right intra- and interhemispheric connectivity during the baseline interval were observed [Figs. 5(a2) versus 5(b2) and 5(c2) versus 5(d2)]. The control group exhibited strong connectivity in right MFG and left MFGright middle temporal gyrus (MTG). Furthermore, connectivity comparisons for the control and disordered groups revealed significances in bilateral intrahemispheric connectivities for the entire measurement [Figs. 5(a1) versus 5(b1) and 5(c1) versus 5(d1)] and task [Figs. 5(a3) versus 5(b3) and 5(c3) versus 5(d3)] intervals. The control group showed stronger right intrahemispheric connectivity than the disordered group; yet, strong left intrahemispheric connectivity was revealed in the disordered group. Statistical results of intergroup (two-sample $t$-test; $p<0.05$ (uncorrected); $\mathrm{DF}=29$ to 57 ; DF depends on each feature availability) connectivity are shown in Fig. S3 in the Supplemental Material. In summary, the strong right intrahemispheric connectivity was consistently observed in the control group during all intervals.

\subsection{Optimization of Feature Selection}

Training (void boxplots) and test (patched boxplots) accuracies during cross-validation (i.e., fivefold) for three ways of selecting features, i.e., best performing single feature (red boxplots), significant between-group features (blue boxplots), and all available features (black boxplots), are compared in Fig. 6. The results imply that combining all available features would not bring better performance compared to selecting fewer features, particularly in training subsets. This trend may be caused by mixing varied feature trends of groups. The significant between-group features used uniform group trends. For example, significantly higher activation features in the control group than in the disordered group were selected. The comparison between performances of the best performing single feature and the significance between-group features were shown to be insignificant in both training and test accuracies. The next question came up: whether the performance of optimized feature selection was superior to the best performing single feature and significant between-group features.

Stepwise training and test accuracies during the optimization of classification analysis are visualized in Fig. 7. There were four points highlighted from the optimization results. First, the training accuracies of activation features were significantly lower compared to connectivity features in all intervals $\left[\mathrm{O}_{2} \mathrm{Hb}\right.$ and $\mathrm{HHb} ; 76 \%$ versus $88 \%$ on average; one-way analysis of variance (ANOVA); $F_{(3,16)}=30.2$ to $33.8 ; \eta^{2}=0.85$ to 0.86 ; $p<0.001]$. Second, there was no significant effect of used features on the test accuracies [one-way ANOVA; $F_{(3,16)}=2.2$ to $\left.2.3 ; \eta^{2}=0.3 ; p>0.05\right]$. The variability of test accuracy evaluated using activation features was relatively high. Third, among connectivity intervals, low training accuracy was observed in $\mathrm{O}_{2} \mathrm{Hb}$ connectivity features during the entire measurement interval [Fig. 7(b1); one-way ANOVA; $F_{(5,24)}=4.2 ; \eta^{2}=0.47$; $p<0.01]$. Fourth, $\mathrm{HHb}$ connectivity features from the entire measurement interval presented higher training and test accuracies than those for $\mathrm{O}_{2} \mathrm{Hb}$ connectivity features [Figs. 7(b1) versus 7(b2); two-sample $t$-test; $t_{(8)}=2.7$ to 7.7; Cohen's $d=1.7$ to $4.9 ; p<0.05$ ]. In general, these results demonstrate the effectiveness of connectivity features in group classification compared to activation features. Although using a great number of features can contribute to better classification performance, high classification analysis using connectivity features was less likely to be caused by the large number of used features (12 to 


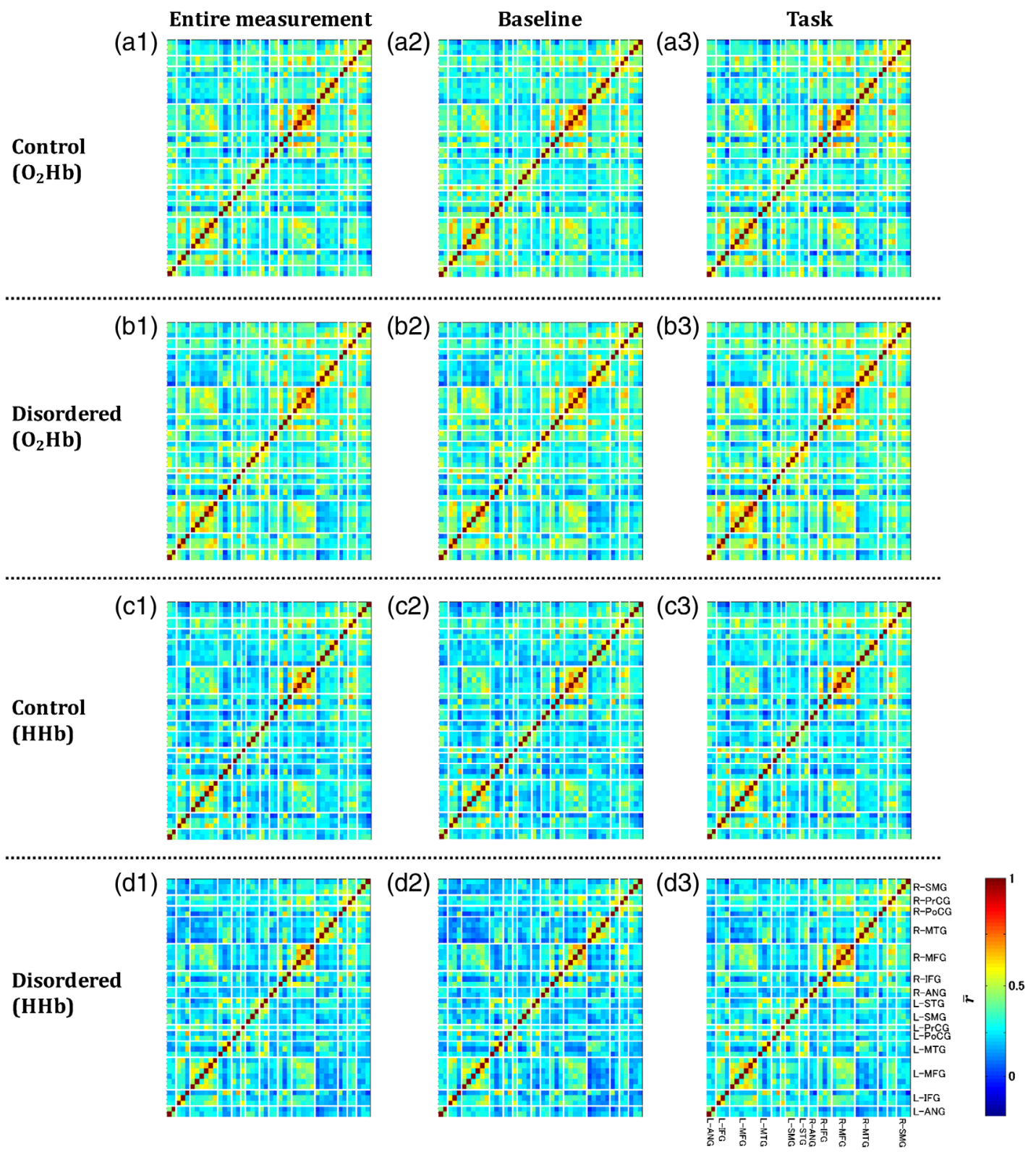

Fig. 5 Channel-wise $\mathrm{O}_{2} \mathrm{Hb}[(\mathrm{a})$ and (b)] and $\mathrm{HHb}[(\mathrm{c})$ and (d)] connectivity maps across subjects $(\bar{r}$, inverse of averaged Fisher- $z$ transform) for control [(a) and (c)] and disordered [(b) and (d)] groups. The connectivity was computed in three intervals: (a1)-(d1) entire measurement, (a2)-(d2) baseline, and (a3)-(d3) task. Channels coming from the same region based on probability of spatial registration are categorized adjacently. Bilateral ( $L$ for left; $R$ for right) cortical areas: ANG, IFG, MFG, MTG, PoCG, precentral gyrus (PrCG), SMG, and STG.

17 versus 8 to 23 features for activation and connectivity, respectively). On the basis of these results, we focused on connectivity features afterward.

To address the question mentioned above, we compare the performance (i.e., training and test accuracies) in terms of best performing single feature, significant between-group features, and optimum features, as shown in Table 1. The optimum features attained significantly higher training accuracies [one-way ANOVA; $F_{(2,12)}=8.28$ to $48.5 ; \eta^{2}=0.58$ to $\left.0.89 ; p<0.01\right]$. No significant benefit of optimum selection was observed in the test accuracy [one-way ANOVA; $F_{(2,12)}=1.2$ to $3.5 ; \eta^{2}=0.17$ to $0.37 ; p>0.05]$. However, the performance of optimum features showed relatively lower standard deviations of test accuracy than those for best performing single feature and significant between-group features (Table 1; red- and blue-patched boxplots in Fig. 6). This result suggests that feature selection is an important step.

\subsection{Performance of Optimum Combination Features}

Features were optimized on the basis of the summation of training and test accuracies. However, well-performing features and classifiers should be able to provide high specificity and sensitivity (i.e., measure of separability). Imbalanced specificity and sensitivity measures could indicate substantial differences in group feature variances. Therefore, we also evaluated specificity and sensitivity in both training and test subsets. As varying 

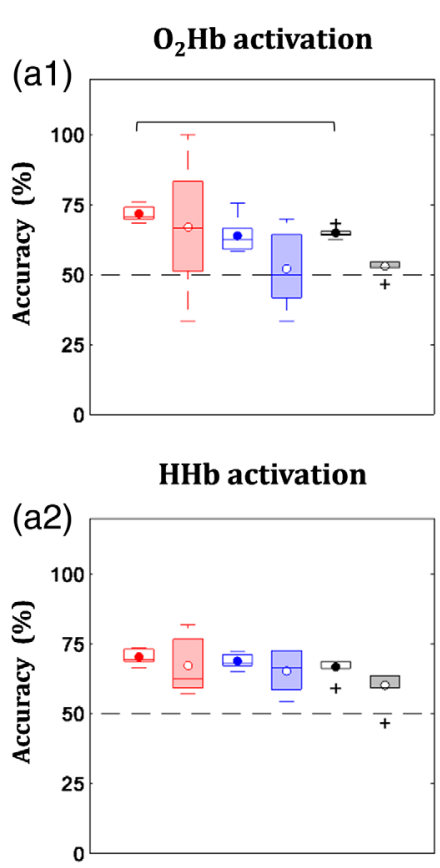

$\mathrm{O}_{2} \mathrm{Hb}$ FC

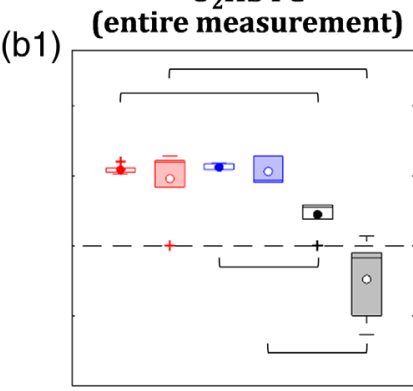

HHb FC

(b2)

[entire measurement]

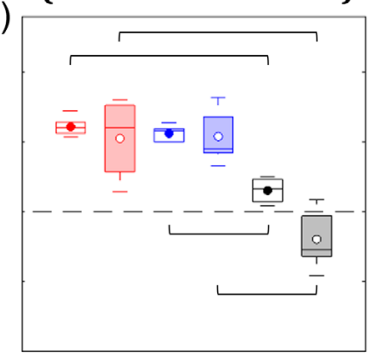

$\mathrm{O}_{2} \mathrm{Hb}$ FC

(c1)

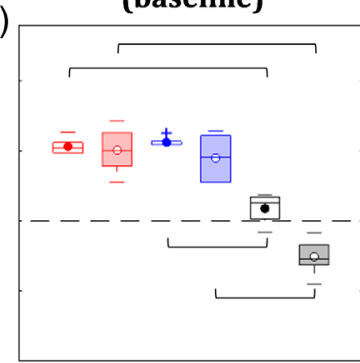

HHb FC

(c2)

(baseline)

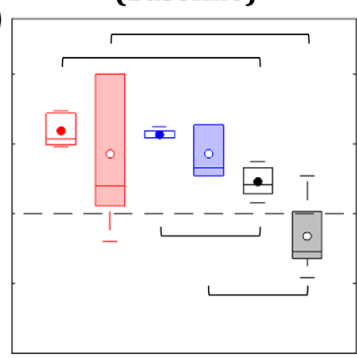

(d1)

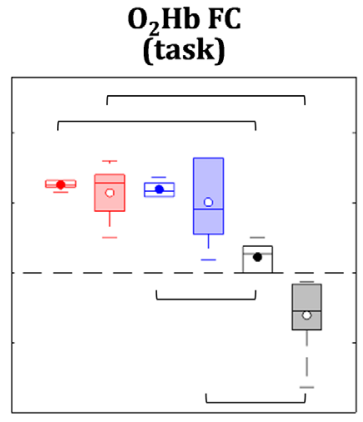

HHb FC (task)

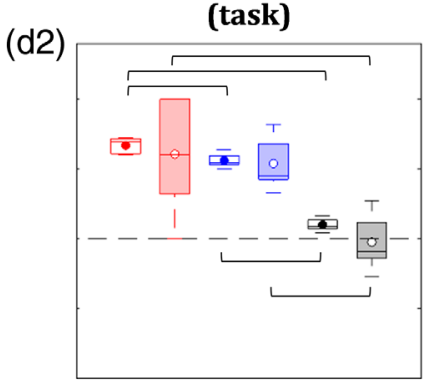

Fig. 6 Comparisons of training (void boxplots) and test (patched boxplots) accuracies during crossvalidation (i.e., fivefold) in the case of three ways of selecting features: best performing single feature (red boxplots), significant between-group features (blue boxplots), and all available features (black boxplots). Comparisons were assessed for (a) activation connectivity features in the (b) entire measurement, (c) baseline, and (d) task intervals for (a1)-(d1) $\mathrm{O}_{2} \mathrm{Hb}$ and (a2)-(d2) $\mathrm{HHb}$. Brackets indicate significant differences between two methods (two-sample $t$-test; $p<0.05$; $\mathrm{DF}=8$ ).

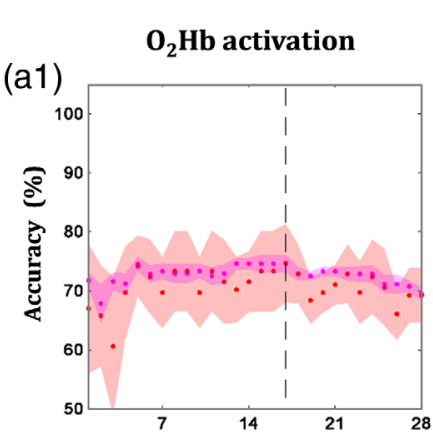

HHb activation

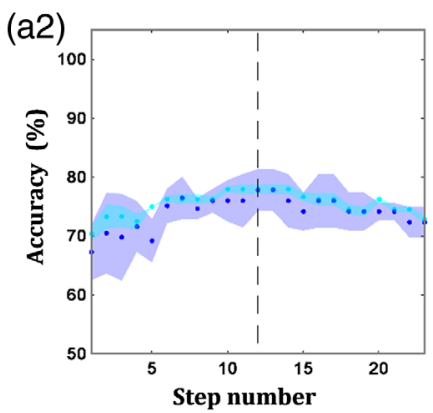

$\mathrm{O}_{2} \mathrm{Hb}$ FC

(b1)

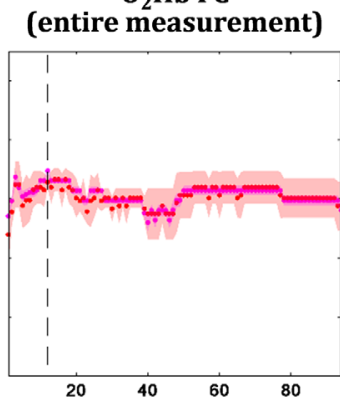

HHb FC

(b2)
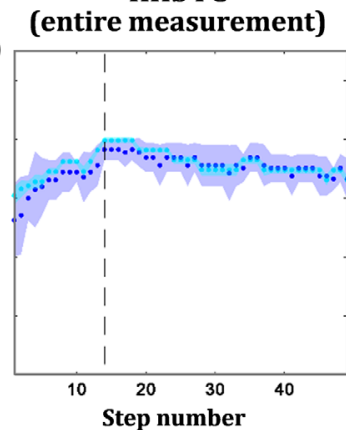

$\mathrm{O}_{2} \mathrm{Hb}$ FC

(c1)

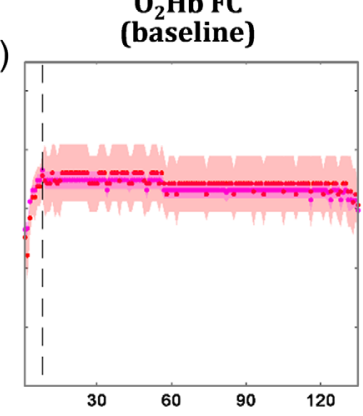

(c2)

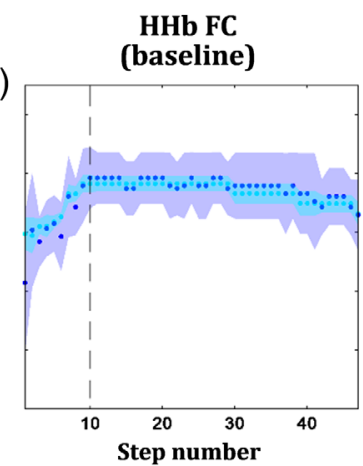

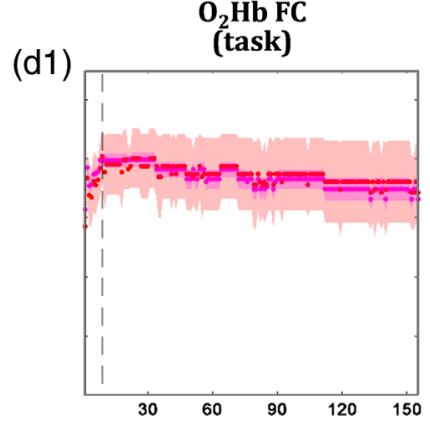

HHb FC

$(\mathrm{d} 2)$

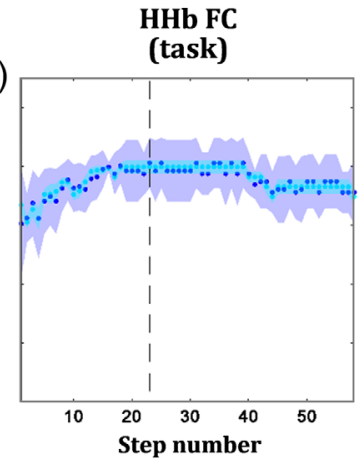

Fig. 7 Stepwise optimization of classification analysis to obtain the highest training (magenta and cyan for $\mathrm{O}_{2} \mathrm{Hb}$ and $\mathrm{HHb}$, respectively) and test (red and blue for $\mathrm{O}_{2} \mathrm{Hb}$ and $\mathrm{HHb}$, respectively) accuracies in (a) activation, connectivity features in the (b) entire measurement, (c) baseline, and (d) task intervals for (a1)-(d1) $\mathrm{O}_{2} \mathrm{Hb}$ and (a2)-(d2) $\mathrm{HHb}$. Scattered points represent the averaged accuracies across cross-validated subsets; neighboring patches are associated with the standard error of accuracies. Dashed lines indicate the highest summation of cross-validated and averaged training and test accuracies. 
Table 1 Training and test performance (mean \pm standard deviation) comparisons of three methods of feature selection (best performing single feature, significant between-group, and stepwise optimization) in $\mathrm{O}_{2} \mathrm{Hb}$ and $\mathrm{HHb}$ connectivities.

\begin{tabular}{|c|c|c|c|c|c|c|}
\hline & \multicolumn{2}{|c|}{ FC (entire measurement) } & \multicolumn{2}{|c|}{ FC (baseline) } & \multicolumn{2}{|c|}{ FC (task) } \\
\hline & $\mathrm{O}_{2} \mathrm{Hb}$ & $\mathrm{HHb}$ & $\mathrm{O}_{2} \mathrm{Hb}$ & $\mathrm{HHb}$ & $\mathrm{O}_{2} \mathrm{Hb}$ & $\mathrm{HHb}$ \\
\hline \multicolumn{7}{|c|}{ Training subsets } \\
\hline Single feature & $77.1 \pm 1.7 \%$ & $80.5 \pm 3.5 \%$ & $76.5 \pm 3.0 \%$ & $79.7 \pm 6.1 \%$ & $81.4 \pm 1.7 \%$ & $83.4 \pm 2.9 \%$ \\
\hline $\begin{array}{l}\text { Significant } \\
\text { features }\end{array}$ & $78.0 \pm 1.3 \%$ & $78.0 \pm 3.0 \%$ & $78.0 \pm 1.8 \%$ & $78.4 \pm 1.8 \%$ & $79.7 \pm 3.0 \%$ & $78.0 \pm 2.6 \%$ \\
\hline \multirow{2}{*}{$\begin{array}{l}\text { Stepwise } \\
\text { selection }\end{array}$} & $84.7 \pm 1.0 \%$ & $89.8 \pm 1.1 \%$ & $86.5 \pm 2.5 \%$ & $88.2 \pm 3.3 \%$ & $89.7 \pm 2.6 \%$ & $89.9 \pm 2.5 \%$ \\
\hline & $\begin{array}{c}F_{(2,12)}=48.5 \\
p<0.001\end{array}$ & $\begin{array}{c}F_{(2,12)}=25.9 \\
p<0.001\end{array}$ & $\begin{array}{c}F_{(2,12)}=24.0 \\
p<0.001\end{array}$ & $\begin{array}{c}F_{(2,12)}=8.28 \\
p<0.01\end{array}$ & $\begin{array}{c}F_{(2,12)}=23.5 \\
p<0.001\end{array}$ & $\begin{array}{c}F_{(2,12)}=24.6 \\
p<0.001\end{array}$ \\
\hline \multicolumn{7}{|l|}{ Test subsets } \\
\hline Single feature & $73.9 \pm 13.5 \%$ & $76.3 \pm 14.0 \%$ & $75.2 \pm 8.4 \%$ & $71.4 \pm 27.2 \%$ & $78.5 \pm 10.4 \%$ & $80.3 \pm 21.1 \%$ \\
\hline $\begin{array}{l}\text { Significant } \\
\text { features }\end{array}$ & $76.5 \pm 4.9 \%$ & $77.0 \pm 9.5 \%$ & $72.4 \pm 8.7 \%$ & $71.5 \pm 9.5 \%$ & $75.2 \pm 15.8 \%$ & $77.0 \pm 9.5 \%$ \\
\hline \multirow{2}{*}{$\begin{array}{l}\text { Stepwise } \\
\text { selection }\end{array}$} & $82.8 \pm 2.2 \%$ & $88.2 \pm 4.0 \%$ & $85.6 \pm 7.9 \%$ & $89.2 \pm 11.7 \%$ & $90.4 \pm 9.6 \%$ & $90.6 \pm 9.6 \%$ \\
\hline & $\begin{array}{c}F_{(2,12)}=1.49 \\
p>0.05\end{array}$ & $\begin{array}{c}F_{(2,12)}=2.24 \\
p>0.05\end{array}$ & $\begin{array}{c}F_{(2,12)}=3.50 \\
p>0.05\end{array}$ & $\begin{array}{c}F_{(2,12)}=1.63 \\
p>0.05\end{array}$ & $\begin{array}{c}F_{(2,12)}=2.13 \\
p>0.05\end{array}$ & $\begin{array}{c}F_{(2,12)}=1.20 \\
p>0.05\end{array}$ \\
\hline
\end{tabular}

thresholds of feature magnitude, averaged receiver operating curves (ROCs) over five times of training are shown in Fig. 8. Results of specificity, sensitivity, and area under ROC are tabulated in Table 2. Among connectivity intervals, the task $\mathrm{HHb}$ connectivity presented the highest specificity [one-way ANOVA; Tukey-Kramer post hoc analysis; $F_{(5,24)}=14.7$; $\eta^{2}=0.75 ; p<0.001$ ] and the lowest sensitivity [one-way ANOVA; Tukey-Kramer post hoc analysis; $F_{(5,24)}=8.2$; $\left.\eta^{2}=0.63 ; p<0.001\right]$. Areas under ROC [one-way ANOVA; $\left.F_{(5,24)}=5.3 ; \eta^{2}=0.52 ; p<0.01\right]$ and differences between specificity and sensitivity [one-way ANOVA; $F_{(5,24)}=4.48$; $\left.\eta^{2}=0.48 ; p<0.01\right]$ were also significantly affected by connectivity intervals. Boxplots of optimum $\mathrm{O}_{2} \mathrm{Hb}$ and $\mathrm{HHb}$ connectivity features are shown in a group-wise manner (Fig. 9). The trends of optimum $\mathrm{O}_{2} \mathrm{Hb}$ and $\mathrm{HHb}$ connectivities were different. Weak $\mathrm{O}_{2} \mathrm{Hb}$ [Figs. 9(b1) and 9(c1)] and strong $\mathrm{HHb}$ [Figs. 9(b2) and 9(c2)] connectivities were observed in the control group. From the training steps, the optimum thresholds of averaged connectivity (inverse of averaged Fisher- $z$ transform) were found to be around 0.2 to 0.3 . Specificity and sensitivity of all connectivity intervals in the test subsets were comparable to each other [one-way ANOVA; $F_{(5,24)}=0.4$ to $1.7 ; \eta^{2}=0.07$ to $0.26 ; p>0.05]$. However, a difference between $\mathrm{O}_{2} \mathrm{Hb}$ and $\mathrm{HHb}$ connectivity performances was found in the entire measurement interval [two-sample $t$-test; $t_{(8)}=2.58$; Cohen's $d=1.6$; $p<0.05]$. According to this result, the use of either baseline (a)

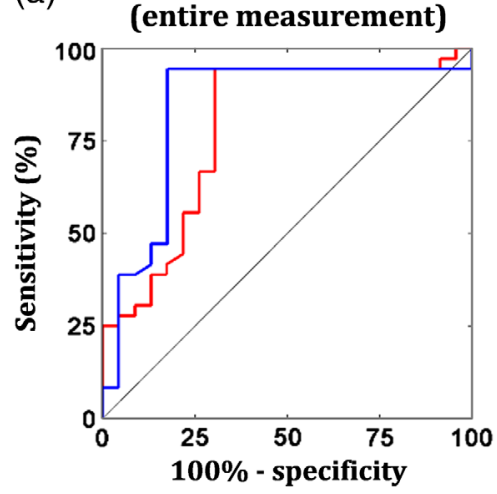

(b)

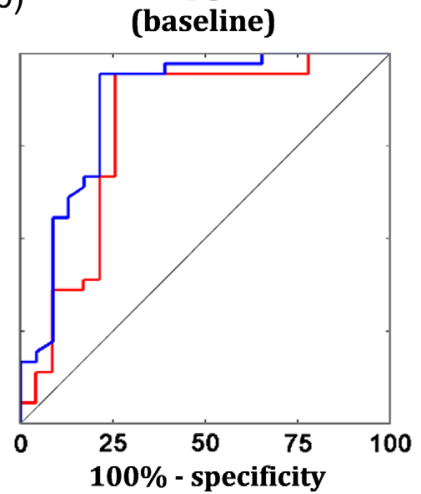

(c)

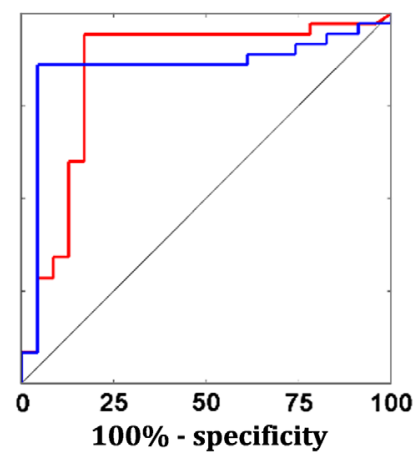

Fig. 8 ROCs denoting specificity and sensitivity of training classification as varying thresholds of optimum $\mathrm{O}_{2} \mathrm{Hb}$ (red curves) and $\mathrm{HHb}$ (blue curves) connectivity features from the (a) entire measurement, (b) baseline, and (c) task intervals. 
Table 2 Training performances of optimum $\mathrm{O}_{2} \mathrm{Hb}$ and $\mathrm{HHb}$ connectivity features from the entire measurement, baseline, and task intervals in terms of specificity, sensitivity, and area under ROC (mean \pm standard deviation).

\begin{tabular}{|c|c|c|c|c|c|c|c|}
\hline & \multicolumn{2}{|c|}{ FC (entire measurement) } & \multicolumn{2}{|c|}{ FC (baseline) } & \multicolumn{2}{|c|}{ FC (task) } & \multirow[b]{2}{*}{ Statistic result } \\
\hline & $\mathrm{O}_{2} \mathrm{Hb}$ & $\mathrm{HHb}$ & $\mathrm{O}_{2} \mathrm{Hb}$ & $\mathrm{HHb}$ & $\mathrm{O}_{2} \mathrm{Hb}$ & $\mathrm{HHb}$ & \\
\hline Specificity & $69.5 \pm 2.3 \%$ & $82.4 \pm 4.1 \%$ & $74.3 \pm 7.8 \%$ & $78.6 \pm 5.7 \%$ & $83.0 \pm 6.5 \%$ & $95.6 \pm 2.5 \%^{a}$ & $F_{(5,24)}=14.7 p<0.001$ \\
\hline Sensitivity & $94.4 \pm 1.9 \%$ & $94.5 \pm 1.9 \%$ & $94.4 \pm 1.9 \%$ & $94.5 \pm 1.9 \%$ & $94.3 \pm 3.2 \%$ & $86.2 \pm 4.1 \%^{\mathrm{a}}$ & $F_{(5,24)}=8.2 p<0.001$ \\
\hline Area under ROC & $78.5 \pm 2.1 \%$ & $83.6 \pm 2.2 \%$ & $79.9 \pm 3.6 \%$ & $86.2 \pm 2.8 \%$ & $84.5 \pm 3.7 \%$ & $85.3 \pm 3.4 \%$ & $F_{(5,24)}=5.3 p<0.01$ \\
\hline
\end{tabular}

${ }^{\mathrm{a}}$ The highest specificity and the lowest sensitivity obtained among all connectivity intervals [one-way ANOVA; Tukey-Kramer post hoc analysis; $F_{(5,24)}=8.2$ to $\left.14.7 ; p<0.001\right]$.
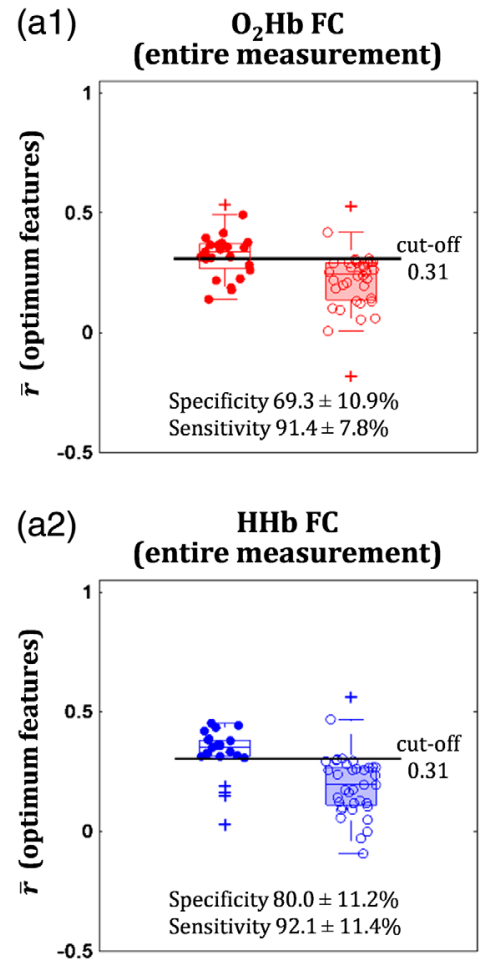
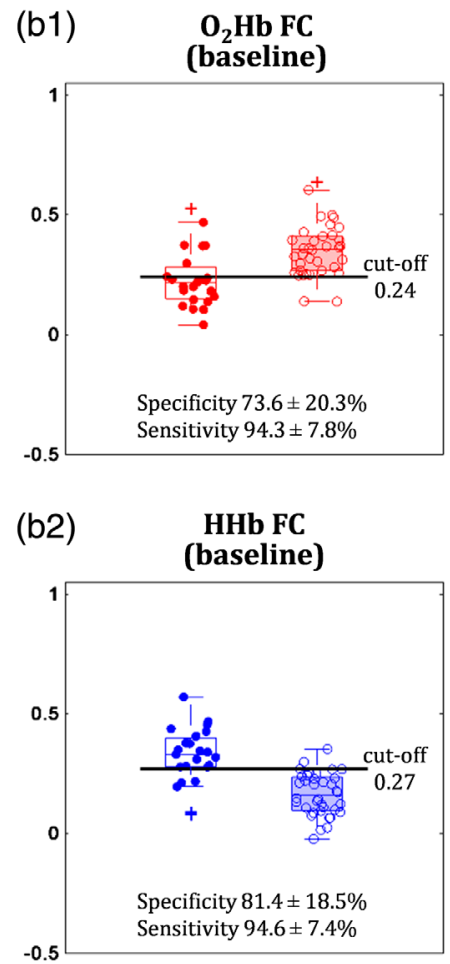
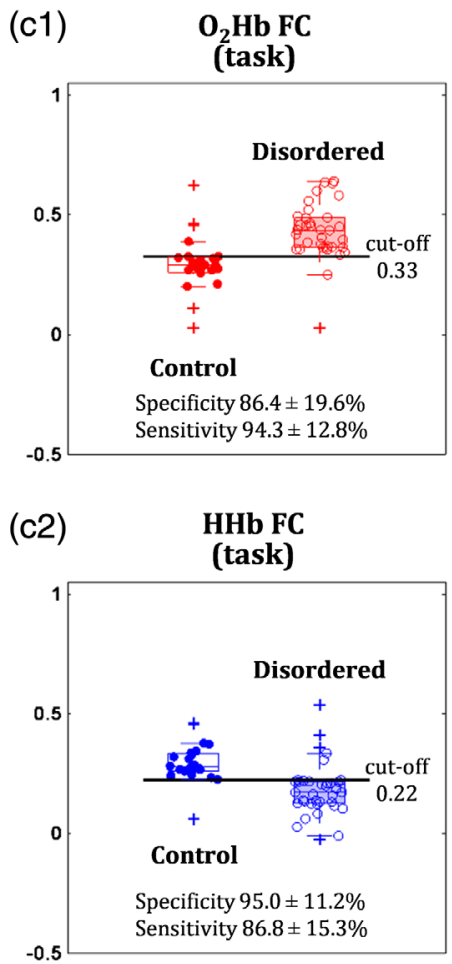

Fig. 9 Distributions of optimum (a1)-(c1) $\mathrm{O}_{2} \mathrm{Hb}$ (red boxplots and scatters) and (a2)-(c2) $\mathrm{HHb}$ (blue boxplots and scatters) classifying features from the connectivities (inverse of averaged Fisher- $z$ transform) of (a) entire measurement, (b) baseline, and (c) task intervals in the control (filled scatters and void boxplots) and disordered (void scatters and patched boxplots) groups. Specificity (true control group; mean \pm standard deviation) and sensitivity (true disordered group; mean \pm standard deviation) values for the test subsets were obtained using the thresholds (i.e., cut-off) optimized in the training subsets.

or task connectivity features $\left(\mathrm{O}_{2} \mathrm{Hb}\right.$ and $\left.\mathrm{HHb}\right)$ for group classification is preferable.

The optimum connectivity features are projected on a brain template in Fig. 10. Both $\mathrm{O}_{2} \mathrm{Hb}$ and $\mathrm{HHb}$ optimum connectivity showed characteristics of interhemispheric connectivity. High $\mathrm{O}_{2} \mathrm{Hb}$ and $\mathrm{HHb}$ connectivities in the control group were clearly denoted by connectivities of right frontal, left frontal, and left parietal lobes [Figs. 10(a), 10(b2), and 10(c2)]. Meanwhile, high $\mathrm{O}_{2} \mathrm{Hb}$ connectivity in the disordered group was observed at connectivities of left frontal, left parietal, and right parietal lobes. In the entire measurement interval, the optimum connectivity nodes of $\mathrm{O}_{2} \mathrm{Hb}$ and $\mathrm{HHb}$ were overlapped [79\% to $91 \%$ overlapped channels; Figs. 10(a1) and 10(a2)] in the right MFG, right MTG, right IFG, right postcentral gyrus (PoCG), left SMG, left superior temporal gyrus (STG), and left ANG.
Meanwhile, mutual connectivity (i.e., vertex) of left ANG (channel 9) with right PoCG (channel 29) and right MTG (channel 30) was observed. This mutual connectivity was also consistently found in optimum $\mathrm{HHb}$ connectivity features from the baseline and task intervals [Figs. 10(a2), 10(b2), and 10(c2)].

\section{Discussion}

The current study evaluated and compared the effectiveness of attention-related brain activation and connectivity features as ADHD (with and without ASD-comorbid) screening biomarkers. Task-based connectivity might be less popular than RS connectivity analysis due to high task demand complicating measurements; however, a symptomatic-related task (e.g., the OB attention task) is likely to be more explainable and controllable, particularly in regard to studying disordered groups. 
(a1) (entire measurement)

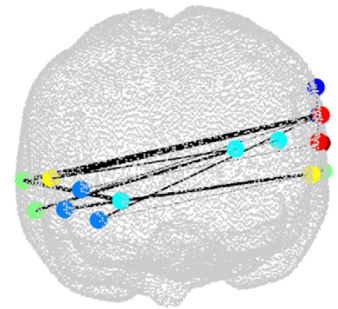

(a2)

(entire measurement)

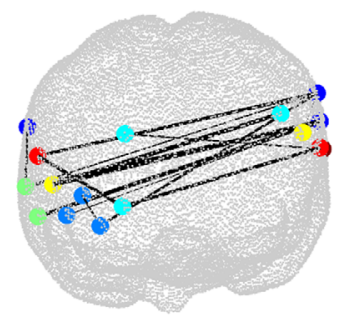

(b1)

$\mathrm{O}_{2} \mathrm{Hb} \mathrm{FC}$ (baseline)

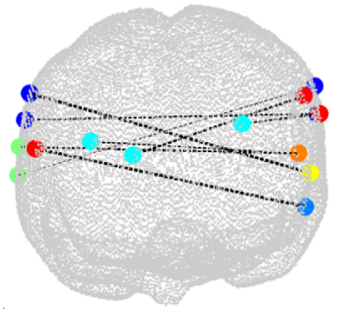

(b2)
HHb FC (baseline)

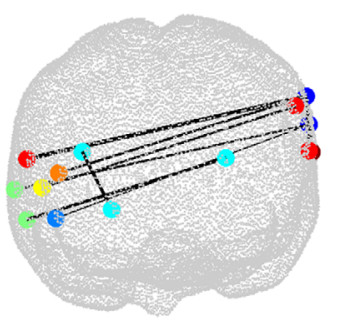

(c1)

$\mathrm{O}_{2} \mathrm{Hb} \mathrm{FC}$

(task)

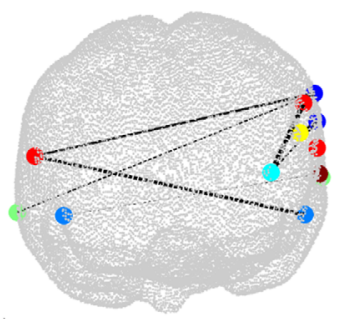

(c2)

HHb FC

(task)

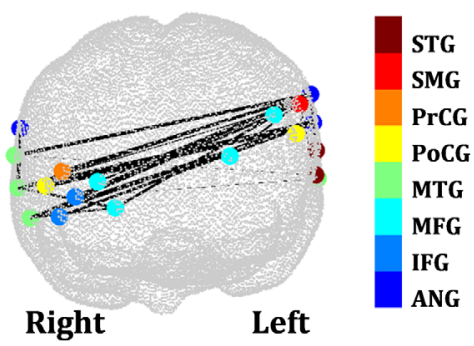

Fig. 10 Optimum (a1)-(c1) $\mathrm{O}_{2} \mathrm{Hb}$ and (a2)-(c2) $\mathrm{HHb}$ classifying features from the connectivities of (a) entire measurement, (b) baseline, and (c) task intervals projected to the brain template. Channel colors are varied according to the region probability such as ANG, IFG, MFG, MTG, PoCG, PrCG, SMG, and STG. Meanwhile, the thickness of connectivity lines indicates $t$-values of control-disordered comparison (two-sample $t$-test). Thicker lines represent stronger probability of control-disordered differences (alternative hypothesis $H_{1}$ : control connectivity is greater than disordered connectivity). Solid connectivity lines [major vertices in (a), (b2), and (c2)] visualize the trend of high connectivity in the control group; dashed-connectivity lines [major vertices in (b1) and (c1)] denote the trend of high connectivity in the disordered group.

Although the activation analysis had been well known and analyzed further as potential biomarkers in ADHD screening ${ }^{52}$ and differential comorbidity diagnosis, ${ }^{49}$ the connectivity analysis unveils an alternative horizon of explaining intra- and interregion relationships. According to our comparison results, the connectivity feature performed well in classifying groups with higher training and test accuracies than the activation features. Combining the practicability of fNIRS, we do hope that the current study contributes to a more accurate and verifiable screening biomarker.

\subsection{Attentive Activation and Connectivity of Disordered Children}

Other modalities, such as electroencephalogram and fMRI, have been used to interpret attentive activation during OB tasks. Human and animal (i.e., monkey) studies on both visual and auditory OB tasks showed transient event-related activation in the bilateral MFG, inferior parietal lobe, and inferior part of the posterior cingulate gyrus. ${ }^{53,54}$ Tamm et al. ${ }^{55}$ investigated the difference in brain activations of healthy and ADHD adolescents measured using fMRI during the OB task. Healthy adolescents exhibited bilateral activation along the frontal (MFG and IFG) to superior parietal (PoCG, SMG, and ANG) lobes as well as posterior cingulate cortex and putamen. Even though the IFG and right anterior/mid-cingulate cortex were activated, those activations were minor compared to those of healthy subjects, and ADHD adolescents had significantly impaired activations on the bilateral parietal lobes, right precuneus, and thalamus. Abnormality of the cingulo-frontal-parietal network in
ADHD patients has also been highlighted. ${ }^{56,57}$ Dysfunctions of the right superior-inferior parietal lobes were also observed in ADHD children during a mental rotation task that required spatial working memory. ${ }^{58}$

Kiehl et al. ${ }^{59}$ demonstrated that spatially diverged activity (i.e., adaptive reflexive processing), which was less likely required for performing a task, was observed even in the case of a low-load task such as the OB task. This finding may correspond to strategic adaptation to any upcoming stimulus. Task-evoked activation of healthy adults found during fMRI measurement was distributed in the bilateral frontal-temporalparietal-occipital lobes of cortical and subcortical systems. The relatively minor activation of ADHD subjects to healthy controls might also imply the disability of adaptive reflexive processing.

We previously reported the results of activation analysis on the currently used datasets. ${ }^{35,40}$ The results revealed impaired activation in the right MFG/IFG and the SMG/ANG for the disordered group. Those ROIs were well aligned with previously reported ones. However, we could not obtain activation information during the baseline interval because the principle of activation analysis is solely comparing between baseline and task-evoked changes. By performing the connectivity analysis for both baseline and task intervals, we found that the high connectivity within the right MFG might not be an OB-evoked response. Within region connectivity of the right MFG had already been strong in the baseline interval. Note that the subjects were required to uniformly and repetitively respond (i.e., press the blue button) to any visual stimulus during the baseline interval. Despite the significant activation increase in the task 
interval, ${ }^{35,40}$ the right $\mathrm{MFG}$ connectivity remained almost unchanged for the control group. This finding suggests that channels within the right MFG were synchronized to focus attention and respond to the visual stimulus; as such, connectivity and activation characteristics might not be positively correlated to each other.

The control group did not show increases of task-evoked connectivity as massive as the disordered group did. This result reveals that the control group relatively maintained the attentive connectivity during baseline and task intervals. According to the obtained task connectivities, task-evoked connectivity of the right MFG was found to increase in the disordered group but not in the control group. However, these connectivity increases were apparently unable to accommodate the lack of brain activation, as previously reported. ${ }^{35,40}$ The disordered group may have the ability to control the attentive connectivity when performing tasks; however, within connectivity of the right MFG (and right intrahemispheric connectivity) were found to be weaker than those for the control group.

The results of hypoactivation and aberrant attention circuitry were consistent with the previous studies-except for the finding of relatively high and increased within region (right MFG) connectivity, which has barely been described in ADHD studies. We argue that the within MFG connectivity might be a result of chronic neuromodulation by stimulant (e.g., MPH and amphetamine) and/or nonstimulant (e.g., ATX) medication. Note that the disordered group was in a premedicated condition; however, none of the subjects were medicated naïve. Long-term MPH administration (i.e., one year) brought improved behavioral performances in the test of variables of attention. ${ }^{60}$ Spencer et al. ${ }^{61}$ reviewed 29 studies related to the psychostimulant effect on the brain and showed the effect of structural and functional alteration suppression relative to unmedicated subjects. However, longitudinal monitoring from medicated naïve status to after treatment is required to verify this argument.

\subsection{Effectiveness of Connectivity Features as a Screening Tool}

Although activation analysis offers information about increased regional blood flow and dismisses temporal information, connectivity analysis identifies functionally synchronized regions with unknown activation significances. Activation and connectivity analyses have advantages and disadvantages; thus combining both analyses will ideally and comprehensively interpret brain activation and networks. The characteristics of activation and networks may potentially be used for screening disordered subjects from TD children.

In the current study, we confirmed the effectiveness of connectivity features as screening biomarkers. We focus on the limited sample number and significant gap in the number of available features: the number of available connectivity features was more than 15 times that of available activation features. When the number of features exceeds the number of samples, a machine learning approach (e.g., a support vector machine) might be more prone to the risk of overfitting. Therefore, we selected a simple threshold-based classification that quantified a combination of features into a single classification parameter (i.e., across features average).

The effectiveness of connectivity features was shown not only in the optimum condition but also in the training performances of a single feature and significant between-group features [Figs. 6(a) versus 6(b), 6(c), 6(d)]. This result suggested that, compared to activation features, the connectivity features are more likely to have prominent group-related information (i.e., high intergroup variance) that contributes to high classification performance. Sole activation features evaluated in medicated naïve ADHD and ASD-comorbid ADHD children were insufficient to construct a differential diagnostic biomarker. ${ }^{49}$ Therefore, we proposed and introduced the MPH-evoked activation as a differential classification feature (accuracy of $82 \%$ ). Nevertheless, administration of medication (even to suspected ADHD children) may be less appropriate, particularly in the case of screening. In light of that perspective, we attempted to develop screening biomarkers on the basis of medication free. The current classification result using unmedicated task connectivity has already been able to offer a classification accuracy of about $88 \%$. This finding has optimized connectivity features in the least burden condition for both control and disordered children.

During the stepwise feature selection, we optimized the use of several connectivities, resulting in the best classification accuracy. Compared to the disordered group, the control group showed stronger connectivities of right frontal, left frontal, and left parietal lobes [Figs. 10(a), 10(b2), and 10(c2)], and revealed weaker connectivities of left frontal, left parietal, and right parietal lobes [Figs. 10(b1) and 10(c1)]. We found that the connectivity between the left ANG (BA 39), right PoCG (BA 3/1/2), and right MTG (BA 21/22) was one of the most robust classification features and had previously been reported to be involved in the attention network. 55,59

In the baseline and task intervals, the optimum $\mathrm{O}_{2} \mathrm{Hb}$ connectivity features were different compared to the optimum $\mathrm{HHb}$ connectivity features [Figs. 10(b1) versus 10(b2) and $10(\mathrm{c} 1)$ versus $10(\mathrm{c} 2)]$. The optimum $\mathrm{O}_{2} \mathrm{Hb}$ connectivity features dominated the group classification based on connectivities of left frontal, left parietal, and right parietal lobes (control $<$ disordered groups); meanwhile, connectivities of right frontal, left frontal, and left parietal lobes (control $>$ disordered groups) characterized the optimum $\mathrm{HHb}$ connectivity features. These differences were caused by the differently selected feature trend at the first optimization step. We manipulated the first selection using the trend of stronger $\mathrm{O}_{2} \mathrm{Hb}$ connectivity in the control group, and we confirmed the similarities between optimum $\mathrm{O}_{2} \mathrm{Hb}$ and $\mathrm{HHb}$ connectivity features (data not shown).

\subsection{Resting State versus Task-Based Connectivity}

Although performing tasks on disordered children heightened the failure risk due to the inability of the children in performing the task, previous studies consequently focused on measurements of children during RS. The feasibility of performing $\mathrm{RS}$ in a great sample number had been confirmed as the completion of an ADHD database from three continents. ${ }^{21}$ The abnormalities on fronto-striatal, fronto-default, and sensorimotor networks were defined as proposed pathophysiological models of ADHD related to the default mode network. ${ }^{62-64}$ Castellanos et al. reported decreased FC between the anterior cingulate and precuneus/posterior cingulate cortices, whereas Tian et al. ${ }^{65}$ oppositely observed significant FC between the anterior cingulate and extensive regions of thalamus, cerebellum, insula, and brainstem in ADHD subjects. These disagreements were likely caused by the analytical reason (i.e., antiphase correlation and seed selection) and divergent maturity level (adolescents versus adults). ${ }^{66}$ Furthermore, symptomatic ADHD severity may influence brain signal variability. ${ }^{67}$ 
According to previous attempts on the development of RS connectivity as a screening tool, the classification performance was unsteady, ranging from $37 \%$ to $86 \% .^{20,21,68}$ The fMRI measurement quality and existence of profound RS connectivity characteristic were then questioned. Therefore, we pursued a different approach on task-based connectivity by controlling the task positive network (i.e., attention network). The current classification result showed superior performance than previously reported classifications on the basis of RS connectivity, despite unmatched symptomatic severity level, comorbidity, and subtype conditions. Thus task-based connectivity features measured using fNIRS may be considered as a substitute for or complement to RS connectivity features for screening purposes.

\subsection{Limitations}

The current study has two limitations. First, the FC-based screening biomarker was optimized and drawn from a small sample number. Due to the small samples, we combined ADHD and ASD-comorbid ADHD children with different acceptabilities of MPH and ATX. In addition, we could not guarantee the absence of overfitting despite our effort to minimize the risk of overfitting. Further verification in the cohort study is necessary. ${ }^{69,70}$ Second, the stepwise feature selection had a drawback of irreversible selection. Validating small test subsets might result in coincidental high performance. Mistakenly selected features even in a step could influence the whole feature combination. Therefore, we also set the training performance as one of the optimization criteria. Even though our current results showed well-performed selection and optimization with high classification accuracy and a robust attention network, mutual information-based methods might be advantageous in regard to extracting profound characteristics with less risk of incorrect selection.

\section{Conclusion}

We investigated attentive task-based connectivity (measured by fNIRS) in control and disordered groups and found that the connectivity showed changes during the transition of baseline-totask, especially in the disordered group. Although the activation of the right MFG was impaired in the disordered group, within region connectivity of the right MFG was found to increase in the task interval. However, intergroup differences were still found, since the disordered group lacked right intrahemispheric connectivity (e.g., within region of MFG) during the baseline and task intervals. The effectiveness of connectivity over activation features for the purpose of a screening biomarker was confirmed. The classification using connectivity features resulted in a high performance with an accuracy of about $88 \%$. With further verification of the effectiveness of connectivity features using a greater number of samples, we expect practical application of task-based connectivity as a robust clinical biomarker.

\section{Disclosures}

The authors have no relevant financial interests in this article and no potential conflicts of interest to disclose.

\section{Acknowledgments}

This work was supported in part by JST-RISTEX to I.D. We would like to thank Dr. Takusige Katura for the fruitful discussion.

\section{References}

1. American Psychiatric Association, Diagnostic and Statistical Manual of Mental Disorders, 5th ed., American Psychiatric Association, Washington (2013).

2. U.S. EPA, "America's Children and the Environment" (2013).

3. S. V. Faraone et al., "The worldwide prevalence of ADHD: is it an American condition?" World Psychiatry 2(2), 104-113 (2003).

4. S. Timimi and E. Taylor, "ADHD is best understood as a cultural construct," Br. J. Psychiatry 184, 8-9 (2004).

5. G. Polanczyk et al., "The worldwide prevalence of ADHD: a systematic review and metaregression analysis," Am. J. Psychiatry 164(6), 942948 (2007).

6. M. Döpfner et al., "How often do children meet ICD-10/DSM-IV criteria of attention deficit-/hyperactivity disorder and hyperkinetic disorder? Parent-based prevalence rates in a national sample-results of the BELLA study," Eur. Child Adolesc. Psychiatry 17(Suppl. 1), 59-70 (2008).

7. C. Adornetto et al., "Concordances and discrepancies between ICD-10 and DSM-IV criteria for anxiety disorders in childhood and adolescence," Child Adolesc. Psychiatry Mental Health 6(40), 1-9 (2012).

8. R. Mayeux, "Biomarkers: potential uses and limitations," NeuroRX 1, 182-188 (2004)

9. P. Boksa, "A way forward for research on biomarkers for psychiatric disorders," J. Psychiatry Neurosci. 38(2), 75-77 (2013).

10. A. Thapar et al., "Advances in genetic findings on attention deficit hyperactivity disorder," Psychol. Med. 37(12), 1681-1692 (2007).

11. M. S. Gold et al., "Low dopamine function in attention deficit/hyperactivity disorder: should genotyping signify early diagnosis in children?," Postgrad. Med. 126(1), 153-177 (2014).

12. O. Kebir et al., "Candidate genes and neuropsychological phenotypes in children with ADHD: review of association studies," J. Psychiatry Neurosci. 34(2), 88-101 (2009).

13. T. Sasaki et al., "Decreased levels of serum oxytocin in pediatric patients with attention deficit/hyperactivity disorder," Psychiatry Res. 228, 746-751 (2015).

14. T. Insel et al., "Research domain criteria (RDoC): toward a new classification framework for research on mental disorders," Am. J. Psychiatry 167(7), 748-751 (2010).

15. B. M. Hager and M. S. Keshavan, "Neuroimaging biomarkers for psychosis," Curr. Behav. Neurosci. Rep. 2, 102-111 (2015).

16. C. H. Y. Fu and S. G. Costafreda, "Neuroimaging-based biomarkers in psychiatry: clinical opportunities of a paradigm shift," Can. J. Psychiatry 58(9), 499-508 (2013).

17. J. W. Bohland et al., "Network, anatomical, and non-imaging measures for the prediction of ADHD diagnosis in individual subjects," Front. Syst. Neurosci. 6, 78 (2012).

18. W. Cheng et al., "Individual classification of ADHD patients by integrating multiscale neuroimaging markers and advanced pattern recognition techniques," Front. Syst. Neurosci. 6, 58 (2012).

19. L. Q. Uddin et al., "Progress and roadblocks in the search for brainbased biomarkers of autism and attention-deficit/hyperactivity disorder," Transl. Psychiatry 7, e1218 (2017).

20. D. Yao et al., "Discriminating ADHD from healthy controls using a novel feature selection method based on relative importance and ensemble learning," in Conf. Proc. IEEE Eng. Med. Biol. Soc., pp. 4632-4635 (2018).

21. The ADHD-200 Consortium, "The ADHD-200 Consortium: a model to advance the translational potential of neuroimaging in clinical neuroscience," Front. Syst. Neurosci. 6, 62 (2012).

22. M. R. G. Brown et al., "ADHD-200 global competition: diagnosing ADHD using personal characteristic data can outperform resting state fMRI measurements," Front. Syst. Neurosci. 6, 69 (2012).

23. S. Durston et al., "Differential patterns of striatal activation in young children with and without ADHD," Biol. Psychiatry 53(10), 871-878 (2003).

24. C. J. Vaidya et al., "Selective effects of methylphenidate in attention deficit hyperactivity disorder: a functional magnetic resonance study," Proc. Natl. Acad. Sci. U. S. A. 95(24), 14494-14499 (1998).

25. D. A. Boas et al., "Twenty years of functional near-infrared spectroscopy: introduction for the special issue," NeuroImage 85, 1-5 (2014).

26. A. Maki et al., "Spatial and temporal analysis of human motor activity using noninvasive NIR topography," Med. Phys. 22(12), 1997-2005 (1995). 
27. L. Duan, Y.-J. Zhang, and C.-Z. Zhu, "Quantitative comparison of resting-state functional connectivity derived from fNIRS and fMRI: a simultaneous recording study," NeuroImage 60(4), 2008-2018 (2012).

28. S. Sasai et al., "A NIRS-fMRI study of resting state network," Neurolmage 63(1), 179-193 (2012).

29. H. Zhang et al., "Test-retest assessment of independent component analysis-derived resting state functional connectivity based on functional near-infrared spectroscopy," NeuroImage 55(2), 607-615 (2011).

30. H. Niu et al., "Test-retest reliability of graph metrics in functional brain networks: a resting-state fNIRS study," PLoS One 8(9), e72425 (2013).

31. H. Niu et al., "Resting-state functional connectivity assessed with two diffuse optical tomographic systems," J. Biomed. Opt. 16(4), 046006 (2011).

32. H. Niu and Y. He, "Resting-state functional brain connectivity: lessons from functional near-infrared spectroscopy," Neuroscientist 20(3), 173188 (2013).

33. S. Lang, N. Duncan, and G. Northoff, "Resting-state functional magnetic resonance imaging: review of neurosurgical applications," Neurosurgery 74(5), 453-465 (2014).

34. Y. Monden et al., "Clinically-oriented monitoring of acute effects of methylphenidate on cerebral hemodynamics in ADHD children using fNIRS," Clin. Neurophysiol. 123(6), 1147-1157 (2012).

35. M. Nagashima et al., "Neuropharmacological effect of methylphenidate on attention network in children with attention deficit hyperactivity disorder during oddball paradigms as assessed using functional near-infrared spectroscopy," Neurophotonics 1(1), 015001 (2014).

36. A. Ishii-Takahashi et al., "Prefrontal activation during inhibitory control measured by near-infrared spectroscopy for differentiating between autism spectrum disorders and attention deficit hyperactivity disorder in adults," NeuroImage 4, 53-63 (2014).

37. T. Matsuo et al., "A preliminary near-infrared spectroscopy study in adolescent and adult patients with attention-deficit/hyperactivity disorder symptoms," Open J. Psychiatry 4, 396-404 (2014).

38. Y. Monden et al., "Right prefrontal activation as a neuro-functional biomarker for monitoring acute effects of methylphenidate in ADHD children: an fNIRS study," NeuroImage 1(1), 131-140 (2012).

39. H. Ichikawa et al., "Hemodynamic response of children with attentiondeficit and hyperactive disorder (ADHD) to emotional facial expressions," Neuropsychologia 63, 51-58 (2014).

40. M. Nagashima et al., "Neuropharmacological effect of atomoxetine on attention network in children with attention deficit hyperactivity disorder during oddball paradigms as assessed using functional near-infrared spectroscopy," Neurophotonics 1(2), 025007 (2014).

41. Z. Li et al., "Dynamic functional connectivity revealed by restingstate functional near-infrared spectroscopy," Biomed. Opt. Express 6(7), 2337-2352 (2015)

42. C. Chang and G. H. Glover, "Time-frequency dynamics of resting-state brain connectivity measured with fMRI," Neurolmage 50(1), 81-98 (2010).

43. D. A. Handwerker et al., "Periodic changes in fMRI connectivity," NeuroImage 63(3), 1712-1719 (2012).

44. S. Sutoko et al., "Adaptive algorithm utilizing acceptance rate for eliminating noisy epochs in block-design functional near-infrared spectroscopy data: application to study in attention deficit/hyperactivity disorder children," Neurophotonics 5(4), 045001 (2018).

45. D. Tsuzuki et al., "Virtual spatial registration of stand-alone fNIRS data to MNI space," NeuroImage 34(4), 1506-1518 (2007).

46. D. Tsuzuki and I. Dan, "Spatial registration for functional near-infrared spectroscopy: from channel position on the scalp to cortical location in individual and group analyses," NeuroImage 85, 92-103 (2014).

47. S. Sutoko et al., "Tutorial on platform for optical topography analysis tools," Neurophotonics 3(1), 010801 (2016).

48. D. T. Delpy et al., "Estimation of optical pathlength through tissue from direct time of flight measurement," Phys. Med. Biol. 33(12), 1433-1442 (1998).

49. S. Sutoko et al., "Distinct methylphenidate-evoked response measured using functional near-infrared spectroscopy during go/no-go task as a supporting differential diagnostic tool between attention-deficit/ hyperactivity disorder and autism spectrum disorder comorbid children," Front. Hum. Neurosci. 13, 7 (2019).

50. D. A. Fair et al., "A method for using blocked and event-related fMRI data to study 'resting state' functional connectivity," NeuroImage 35(1), 396-405 (2007).
51. R. R. Hocking, "The analysis and selection of variables in linear regression," Biometrics 32, 1-49 (1976).

52. Y. Monden et al., "Individual classification of ADHD children by right prefrontal hemodynamic responses during a go/no-go task as assessed by fNIRS," Neurolmage 9, 1-12 (2015).

53. K. A. Paller et al., "Potentials evoked in human and monkey medial temporal lobe during auditory and visual oddball paradigms," Electroencephalogr. Clin. Neurophysiol. 84, 269-279 (1992).

54. G. McCarthy et al., "Infrequent events transiently activate human prefrontal and parietal cortex as measured by functional MRI," J. Neurophysiol. 77, 1630-1634 (1997).

55. L. Tamm, V. Menon, and A. L. Reiss, "Parietal attentional system aberration during target detection in adolescents with attention deficit hyperactivity disorder: event-related fMRI evidence," Am. J. Psychiatry 163, 1033-1043 (2006).

56. G. Bush, "Attention-deficit/hyperactivity disorder and attention networks," Neuropsychopharmacol. Rev. 35, 278-300 (2010).

57. G. Bush, "Cingulate, frontal and parietal cortical dysfunction in attention-deficit/hyperactivity disorder," Biol. Psychiatry 69(12), 1160-1167 (2011).

58. A. Vance et al., "Right parietal dysfunction in children with attention deficit hyperactivity disorder, combined type: a functional MRI study," Mol. Psychiatry 12, 826-832 (2007).

59. K. A. Kiehl et al., "An adaptive reflexive processing model of neurocognitive function: supporting evidence from a large scale $(\mathrm{n}=100)$ fMRI study of an auditory oddball task," NeuroImage 25(3), 899915 (2005).

60. Y.-S. Huang, L.-J. Wang, and C.-K. Chen, "Long-term neurocognitive effects of methylphenidate in patients with attention deficit hyperactivity disorder, even at drug-free status," BMC Psychiatry 12, 194 (2012).

61. T. Spencer et al., "Effect of psychostimulants on brain structure and function in ADHD: a qualitative literature review of MRI-based neuroimaging studies," J. Clin. Psychiatry 74(9), 902-917 (2013).

62. F. X. Castellanos et al., "Cingulate-precuneus interactions: a new locus of dysfunction in adult attention-deficit/hyperactivity disorder," Biol. Psychiatry 63(3), 332-337 (2008).

63. F. X. Castellanos et al., "Characterizing cognition in ADHD: beyond executive dysfunction," Trends Cognit. Sci. 10(3), 117-123 (2006).

64. J. Choi et al., "Aberrant development of functional connectivity among resting state-related functional networks in medication-naive ADHD children," PLoS One 8(12), e83516 (2013).

65. L. Tian et al., "Altered resting-state functional connectivity patterns of anterior cingulate cortex in adolescents with attention deficit hyperactivity disorder," Neurosci. Lett. 400(1-2), 39-43 (2006).

66. F. X. Castellanos, A. M. C. Kelly, and M. P. Milham, "The restless brain: attention-deficit/hyperactivity disorder, resting state functional connectivity and intrasubject variability," Can. J. Psychiatry 54(10), 665-672 (2009).

67. J. S. Nomi et al., "Resting-state brain signal variability in prefrontal cortex is associated with ADHD symptom severity in children," Front. Hum. Neurosci. 12, 90 (2018).

68. A. D. S. Siquera et al., "Abnormal functional resting-state networks in ADHD: graph theory and pattern recognition analysis of fMRI data," BioMed. Res. Int. 2014, 1-10 (2014).

69. K. Linnet et al., "Quantifying the accuracy of a diagnostic test or marker," Clin. Chem. 58(9), 1292-1301 (2012).

70. P. M. M. Bossuyt et al., "Beyond diagnostic accuracy: the clinical utility of diagnostic tests," Clin. Chem. 58(12), 1636-1643 (2012).

Stephanie Sutoko received her bachelor's degree in engineering from Bandung Institute of Technology, Indonesia, in 2011 and her master's degree from the University of Tokyo, Japan, in 2014. She started her career as a researcher at the Center for Exploratory Research, Research and Development (R\&D) Group, Hitachi, Ltd., Japan, in 2014. She is also currently pursuing a doctoral degree from Chuo University, Japan. Her research interests include NIRS applications in psychiatric, signal processing, and data analysis.

Yukifumi Monden received his medical degree in Japan in 2002 and his PhD from Jichi Medical University, Japan, in 2013. His major professional achievements include being the chief intern at the U.S. Naval Hospital, Okinawa, Japan, from 2006 to 2007 and a clinical associate from 2009 to 2012 . He is currently an assistant professor 
in the Department of Pediatrics at Jichi Medical University and has been a professor in the Department of Pediatrics, International University of Health and Welfare, Japan (since 2018). His research focuses on neuroimaging studies of neurodevelopmental disorders.

Tatsuya Tokuda is a master's student at Chuo University, Japan. He has been engaged in fNIRS research since 2015. He has authored seven peer-reviewed articles. His research focuses on clinical applications of fNIRS.

Takahiro Ikeda received his medical degree in Japan in 2007 and his PhD from Jichi Medical University, Japan, in 2019. He was a resident from 2007 to 2012 and has been a research associate in the Department of Pediatrics, Jichi Medical University, Japan, since 2012. He was also a part of the medical staff at the International University of Health and Welfare Rehabilitation Center, Nasu Institute for Developmental Disabilities, Japan, from 2012 to 2014. His research focuses on neuroimaging studies of neurodevelopmental disorders using fNIRS.

Masako Nagashima received her medical degree in Japan in 2004 She was a resident from 2004 to 2009 and has been a research associate in the Department of Pediatrics, Jichi Medical University, Japan, since 2009. She was also a chief of inpatient medicine at the International University of Health and Welfare, Japan, from 2012 to 2013. She was a guest researcher at the University of Tübingen, Germany, from 2017 to 2019 . Her research focuses on neuroimaging studies of neurodevelopmental disorders.

Tsukasa Funane received his bachelor's degree in engineering from the University of Tokyo, Japan, in 2004 and his $\mathrm{PhD}$ in engineering from Keio University, Japan, in 2012. He is a senior researcher at R\&D Group, Hitachi, Ltd., Japan. He has been a member of the research group working on optical brain function monitoring technology based on NIRS. His research interests include biomedical optics and its neuroscience and medical applications. $\mathrm{He}$ is a member of SPIE.

Hiroki Sato received his master's degree from the University of Tokyo, Japan, in 2000 and his PhD from Keio University, Japan, in 2006. He was a senior researcher at R\&D Group, Hitachi, Ltd.,
Japan from 2011 to 2018 and was a guest researcher at the University of Tübingen, Germany, from 2013 to 2014 . Currently, he is a professor at Shibaura Institute of Technology, Japan. His research interests include cognitive neuroscience and fNIRS applications.

Masashi Kiguchi is a senior researcher at R\&D Group, Hitachi, Ltd., Japan. His background is in physics and laser spectroscopy. He is interested in fundamental problems of NIRS technique. He has been taking the lead to develop an innovative brain measurement technique in developing alternative research fields and its practical uses.

Atsushi Maki received his $\mathrm{PhD}$ in medical engineering. He joined Hitachi, Ltd., R\&D Group in 1990. He was an invited researcher at the University College London in 2002. He developed a new neuroimaging method, namely, optical topography in 1995. Currently, he engages in works related to the developmental human science and complex brain diseases. His research area is developing solutions for societal issues based on human and brain measurements (e.g., neuropharmacology, diagnosis of brain disease, and neurodesign for industrial areas).

Takanori Yamagata received his medical degree in Japan in 1986 and his PhD from Jichi Medical University, Japan, in 1993. He was a research associate in the Department of Molecular and Human Genetics at Baylor College of Medicine, from 1997 to 2000 . He is currently a professor in the Department of Pediatrics, Jichi Medical University, Japan (since 2011). His research interests include studies on the molecular pathogenesis of autism spectrum disorder, one of the neurodevelopmental disorders.

Ippeita Dan received his $\mathrm{PhD}$ from the University of Tokyo, Japan, in 2002. He was a senior research fellow at the National Food Research Institute and an associate professor at Jichi Medical University, Japan. He was appointed as a professor at Chuo University, Japan. $\mathrm{He}$ has authored more than 100 peer-reviewed articles attracting about 7000 citations. His research missions lie in clinical applications of fNIRS, methodological development of fNIRS data analyses, and psychometrics applications for business marketing (e.g., food manufacturing). He has served as a director on the board of the Society of fNIRS since 2016. 